\title{
NEMATIC ELASTOMERS: GAMMA-LIMITS FOR LARGE BODIES AND SMALL PARTICLES*
}

\author{
PIERLUIGI CESANA ${ }^{\dagger}$
}

\begin{abstract}
We compute the large-body and the small-particle Gamma-limit of a family of energies for nematic elastomers. We work under the assumption of small deformations (linearized kinematics) and consider both compressible and incompressible materials. In the large-body asymptotics, even if we describe the local orientation of the liquid crystal molecules according to the model of perfect order (Frank theory), we prove that we obtain a fully biaxial nematic texture (that of the de Gennes theory) as a by-product of the relaxation phenomenon connected to Gamma-convergence. In the case of small particles, we show that formation of new microstructure is not possible, and we describe the map of minimizers of the Gamma-limit as the phase diagram of the mechanical model.
\end{abstract}

Key words. nematic liquid crystal elastomers, linearized elasticity, Gamma-convergence

AMS subject classifications. 74B05, 49J45, 49S05, 82D30

DOI. $10.1137 / 100795619$

1. Introduction. Studying the microstructure of complex materials is one of the most interesting problems in modern applied mathematics and statistical mechanics. A paradigmatic case is represented by nematic liquid crystal elastomers (LCEs), a class of materials which associate a liquid crystalline texture composed of rigid rod-like molecules (nematic mesogens) with an elastic medium. To sketch the internal organization of this material, we recall that the backbone of the elastomer is constituted by long polymeric chains which are cross-linked to a substrate. Then rod-like molecules of a nematic liquid crystal are linked to the chains. As a result, the topology of the mesogens is fixed and a mechanical deformation can reorient locally the nematic molecules and modify the optical properties of the elastomer. Their interesting properties stem from the interaction between liquid crystalline order and the elastic response of the chains. A very relevant phenomenon observed in nematic elastomers is the large spontaneous deformation accompanying a temperature-induced phase transformation from the isotropic to the nematic state. This deformation can reach $400 \%$ with respect to the reference configuration. Moreover, LCEs can deform and bend under UV-light excitation or in the presence of electric or magnetic fields. These properties make them extremely interesting for applications in bioengineering and robotics (e.g., artificial muscles and crystalline).

This paper is part of a series of articles concerning the analysis of functionals for LCEs in the scenario of the linearized elasticity. Our main reference on the mathematical theory of elasticity is [10]. We refer to [15], [28], and [29] for a physical and mathematical introduction to liquid crystals and liquid crystal elastomers. The general approach to modeling the nematic mesogens is to define an order tensor [15], [28].

*Received by the editors May 17, 2010; accepted for publication (in revised form) June 21, 2011; published electronically October 20, 2011. This work was partially supported by project MTM200907662 of the Spanish Ministry of Science and Innovation and by the Department of Energy - National Nuclear Security Administration under award DE-FC5208NA28613 through Caltech's PSAAP Center for the Predictive Modeling and Simulation of High Energy Density Dynamic Response of Materials. http://www.siam.org/journals/sima/43-5/79561.html

†SISSA-International School for Advanced Studies, Trieste, Italy, and BCAM, Basque Center for Mathematics, Derio, Spain. Current address: California Institute of Technology, Pasadena, CA (cesana@caltech.edu). 
In brief, the information on the orientation and the degree of order of the nematic molecules is encoded in the eigenvectors and in the eigenvalues of the symmetric matrix $\mathbf{Q}$, a state variable which can be defined in three different ways according to the three available models of de Gennes, Ericksen, and Frank. Though the geometrically linear theory has some obvious limitations, it is a very valuable conceptual tool in the study of phase transforming materials. In particular, it lends itself more easily to the exploration of model extensions such as, for example, accounting for the presence of electric or magnetic fields (see [8]) and including curvature elasticity terms typical of liquid crystals by simply adding new terms in the governing energy. Furthermore, the linear theory is simpler in many respects and, importantly, the resulting energy landscapes have an easier geometric structure (see also [7] and [9]). Therefore, rigorous mathematical results such as, for example, the explicit construction of the relaxed energies are more complete, leading to a great insight into the energetically optimal states of the material.

Denoting by $\mathbf{F}$ the gradient of the displacement, a possible expression for the energy density describing nematic elastomers is [8]

$$
f_{m e c}(\mathbf{Q}, \mathbf{F})=\mu\left|\left(\frac{\mathbf{F}+\mathbf{F}^{T}}{2}\right)-\gamma \mathbf{Q}\right|^{2}+\frac{\lambda}{2}(\operatorname{tr} \mathbf{F})^{2} .
$$

The order tensor $\mathbf{Q}$ can be taken either in $\mathcal{Q}_{F r}$, or $\mathcal{Q}_{U}$, or $\mathcal{Q}_{B}$ (respectively, the set of Frank, Ericksen, and de Gennes order tensors). The relationship between this expression and the one proposed in the Cambridge group [29] within the framework of finite elasticity is discussed in [1] and [20]. The sets $\mathcal{Q}_{F r} \subset \mathcal{Q}_{U} \subset \mathcal{Q}_{B}$ are defined in section 1.1. Here we recall that the theory of de Gennes [15] allows us to describe both the degree of nematic order (including biaxiality) and the average direction of the molecules. In particular, the convex and compact set $\mathcal{Q}_{B}$ contains biaxial matrices and, among them, the null element. A simplified theory can be obtained by ruling out biaxiality from the available states of order, in which case we obtain the Ericksen model of uniaxial tensors [21]. A further simplification consists in considering the order of the system as frozen, so that the only unknown parameter describing nematic mesogens is their local orientation. This is the model of perfect order, or the Frank model [23]. It is noticeable that both $\mathcal{Q}_{U}$ and $\mathcal{Q}_{F r}$ are nonconvex sets, and that $\mathcal{Q}_{U}$ contains the null tensor, while $\mathcal{Q}_{F r}$ does not. Therefore, in the case in which we take $\mathbf{Q} \in \mathcal{Q}_{F r}$ in (1.1) a state with biaxial or zero $\mathbf{Q}$ cannot be induced mechanically, while if $\mathbf{Q} \in \mathcal{Q}_{U}$, the isotropic state (i.e., $\mathbf{Q}=0$ ) is reachable. On the other hand, if $\mathbf{Q} \in \mathcal{Q}_{B}$, we can obtain all the biaxial states, including again $\mathrm{Q}=0$.

The considerations above pertain to the microscopic description of order in nematic elastomers. In fact, in stretching experiments of sufficiently large samples, loss of local order and isotropy can be observed even on a macroscopic scale. The former is the formation of stripe-domains, and the second is blurring near the clamps. These phenomena can be described by the analysis of the large-body Gamma-limit of functionals defined in the scenario of the Frank tensor. More precisely, all of these material instabilities are modeled by low energy minimizing sequences for functionals defined over nonclosed sets of functions. On the other hand, if we apply a deformation to a very small sample of nematic elastomers, we observe a uniform orientation of the mesogens. In this case, formation of microstructure seems to be ruled out.

This paper concerns the asymptotic analysis of minima and minimizers of the 
integral functional for incompressible nematic elastomers:

$$
(\mathbf{Q}, \mathbf{u}) \mapsto \begin{cases}\int_{\Omega}\left(\varepsilon^{2}|\nabla \mathbf{Q}|^{2}+f_{\text {mec }}(\mathbf{Q}, \nabla \mathbf{u})\right) d x \\ \quad \text { on } H^{1}\left(\Omega, \mathcal{Q}_{X}\right) \times H_{o}^{1}\left(\Omega, \mathbb{R}^{3}\right), \operatorname{div} \mathbf{u}=0, \\ +\infty \quad \text { otherwise in } L^{2}\left(\Omega, \mathbb{M}^{3 \times 3}\right) \times H^{1}\left(\Omega, \mathbb{R}^{3}\right),\end{cases}
$$

where $\mathbf{u}$ is the mechanical displacement, $\Omega$ is a suitable domain in $\mathbb{R}^{3}$, and $X$ is a label which stands either for $F r$, or $U$, or $B$, according to the available models of order tensors. The parameter $\varepsilon$ is proportional to the ratio between the curvature constant of the liquid crystal and the characteristic length of the specimen. In brief, in the large-body limit $\varepsilon$ tends to zero, while in the small-particle limit $\varepsilon$ tends to infinity. We compute exactly the two variational limits of (1.2) as $\varepsilon \rightarrow 0$ and as $\varepsilon \rightarrow \infty$ in the sense of Gamma-convergence with respect to suitable topologies. Our main result (Theorem 3) is the computation of the large-body Gamma-limit in all three cases (i.e., when $X$ stands for either $F r$, or $U$, or $B$ ), which is

$$
(\mathbf{Q}, \mathbf{u}) \mapsto\left\{\begin{array}{cc}
\int_{\Omega} f_{m e c}(\mathbf{Q}, \nabla \mathbf{u}) d x & \text { on } L^{2}\left(\Omega, \mathcal{Q}_{B}\right) \times H_{o}^{1}\left(\Omega, \mathbb{R}^{3}\right), \operatorname{div} \mathbf{u}=0 \\
+\infty & \text { otherwise in } L^{2}\left(\Omega, \mathbb{M}^{3 \times 3}\right) \times H^{1}\left(\Omega, \mathbb{R}^{3}\right) .
\end{array}\right.
$$

This result is straightforward in the case in which we set $X=B$ in (1.2), since the limit energy reduces in fact to the integral of the density $f_{m e c}$, and there is no penalization for the gradient of the order tensor. A very interesting fact is that the energy appearing in (1.3) is the Gamma-limit of (1.2) even if we assume $X=F r$ (or $X=U$ ), that is, if we model the crystal according to the Frank (or the uniaxial) order tensor theory. Therefore, a biaxial texture can be obtained effectively thanks to the formation of microstructure, even though biaxiality is excluded at the microscopic level. We anticipate that this Gamma-convergence result is based on the fact that the subset of Frank tensors $L^{2}\left(\Omega, \mathcal{Q}_{F r}\right)$ is not weakly closed, and that its closed convex hull coincides with $L^{2}\left(\Omega, \mathcal{Q}_{B}\right)$. The physical consequences of this result are deep and are discussed in the rest of the article. Furthermore, in the converse regime, namely, the small-particle asymptotics, we show that the Gamma-limit of (1.2) reduces to the integral of $f_{m e c}$ defined over the set of constant order tensors, that is,

$$
(\mathbf{Q}, \mathbf{u}) \mapsto\left\{\begin{array}{cc}
\int_{\Omega} f_{\text {mec }}(\mathbf{Q}, \nabla \mathbf{u}) d x & \text { on }\left\{H^{1}\left(\Omega, \mathcal{Q}_{X}\right), \text { Const }\right\} \times H_{o}^{1}\left(\Omega, \mathbb{R}^{3}\right), \operatorname{div} \mathbf{u}=0 \\
+\infty & \text { otherwise in } L^{2}\left(\Omega, \mathbb{M}^{3 \times 3}\right) \times H^{1}\left(\Omega, \mathbb{R}^{3}\right) .
\end{array}\right.
$$

The rest of the paper is organized as follows. In the remaining subsections of the introduction we present the form of the energy functional and its rescaling. Section 2 is devoted to the analysis of large bodies. For the reader's convenience, the proof of the Gamma-convergence is subdivided into two main parts, the first one (section 2.1) concerning the relaxation (i.e., the computation of the lower semicontinuous envelope) of the mechanical energy, and the second one (section 2.2) containing the Gamma-limit argument. Then in section 2.3 we compute some special equilibrium solutions and compare the Gamma-convergence results of this paper with the relaxation of another model [7] which was introduced to describe microstructure formation in nematic elastomers. Finally, section 3 contains the analysis for small particles. All the Gamma-convergence results are obtained also for models of compressible materials (in other words, if we remove the constraint on the divergence of $\mathbf{u}$ ) and in the presence of slightly different boundary conditions, in order to describe a variety of physical problems. 


\subsection{Preliminaries.}

Notation. We gather here the main symbols and the notation used throughout the paper. Let $\mathbb{N}$ and $\mathbb{R}$ denote the set of natural and real numbers, respectively. For any integer $n, \mathbb{R}^{n}$ is the space of $n$-dimensional vectors with canonical basis $\left\{\mathbf{i}_{i}\right\}, i=$ $1, \ldots, n$, with origin $O=(0, \ldots, 0)$ and $\mathbb{M}^{n \times n}$ the space of square real matrices. The determinant, the trace, and the transpose of the matrix $\mathbf{F}$ in $\mathbb{M}^{n \times n}$ are denoted by $\operatorname{det} \mathbf{F}, \operatorname{tr} \mathbf{F}, \mathbf{F}^{T}$, respectively. We endow $\mathbb{M}^{n \times n}$ with the usual inner product $\mathbf{F}: \mathbf{M}:=\operatorname{tr}\left(\mathbf{F M}^{T}\right)=\sum_{i j} F_{i j} M_{i j}$. Here $M_{i j}, F_{i j}$ are the cartesian components of $\mathbf{M}$ and $\mathbf{F}$. The identity in $\mathbb{M}^{n \times n}$ is denoted by $\mathbf{I}$. Focusing on the case $n=$ 3 , we label with $\mathbb{M}_{0}^{3 \times 3}, \mathbb{M}_{\text {sym }}^{3 \times 3}$, and $\mathbb{M}_{0 s y m}^{3 \times 3}$ the subspaces of traceless, symmetric, and traceless and symmetric (deviatoric) matrices, respectively. We introduce the projections $\mathbb{E}: \mathbb{M}^{3 \times 3} \mapsto \mathbb{M}_{\text {sym }}^{3 \times 3}$, defined as $\mathbb{E}(\mathbf{F}):=\left(\mathbf{F}+\mathbf{F}^{T}\right) / 2$, and $\mathbb{E}_{0}: \mathbb{M}^{3 \times 3} \mapsto$ $\mathbb{M}_{0 \text { sym }}^{3 \times 3}$, defined as $\mathbb{E}_{0}(\mathbf{F}):=\mathbb{E}(\mathbf{F})-((\operatorname{tr} \mathbf{F}) / 3) \mathbf{I}$. In this definition we can consider the trace of $\mathbb{E}(\mathbf{F})$ as well, since $\operatorname{tr} \mathbf{F}=\operatorname{tr} \mathbb{E}(\mathbf{F})$. This fact will be widely used in what follows. If we introduce $\mathbf{F}^{s k}:=\left(\mathbf{F}-\mathbf{F}^{T}\right) / 2$, we obtain the well-known decomposition $\mathbf{F}=\mathbb{E}_{0}(\mathbf{F})+\mathbf{F}^{s k}+\frac{(\operatorname{tr} \mathbf{F})}{3} \mathbf{I}$. We denote by $\mathbb{C}: \mathbb{M}_{\text {sym }}^{3 \times 3} \mapsto \mathbb{M}_{\text {sym }}^{3 \times 3}$ the fourth-order tensor of linearized isotropic elasticity, given as $\mathbb{C}(\mathbf{A})=2 \mu \mathbf{A}+\lambda \operatorname{tr}(\mathbf{A}) \mathbf{I} \forall \mathbf{A} \in \mathbb{M}_{\text {sym }}^{3 \times 3}$, with positive $\mu$ and $\lambda$ (Lamé constants).

Definitions and properties of the sets $\mathcal{Q}_{B}, \mathcal{Q}_{U}, \mathcal{Q}_{F r}$. We define the set of biaxial (de Gennes) tensors

$$
\mathcal{Q}_{B}:=\left\{\mathbf{Q} \in \mathbb{M}_{\text {sym }}^{3 \times 3}: \operatorname{tr} \mathbf{Q}=0,-\frac{1}{3} \leq \lambda_{\min }(\mathbf{Q}) \leq \lambda_{\max }(\mathbf{Q}) \leq \frac{2}{3}\right\},
$$

where $\lambda_{\min }(\mathbf{Q})$ and $\lambda_{\max }(\mathbf{Q})$ denote the smallest and largest eigenvalue of the matrix $\mathbf{Q}$. We prove that $\mathcal{Q}_{B}$ is convex. To this end, we recall that $\mathbf{Q} \mapsto \lambda_{\min }(\mathbf{Q})$ is a concave and continuous function, and $\mathbf{Q} \mapsto \lambda_{\max }(\mathbf{Q})$ is a convex and continuous function [25, section 5.1, Theorem 3.1.1]. Hence, if we take $\mathbf{Q}_{1}, \mathbf{Q}_{2} \in \mathcal{Q}_{B}$ and any $\nu \in[0,1]$, we have

$$
\lambda_{\min }\left(\nu \mathbf{Q}_{1}+(1-\nu) \mathbf{Q}_{2}\right) \geq \nu \lambda_{\min }\left(\mathbf{Q}_{1}\right)+(1-\nu) \lambda_{\min }\left(\mathbf{Q}_{2}\right) \geq-1 / 3
$$

and, analogously, $\lambda_{\max }\left(\nu \mathbf{Q}_{1}+(1-\nu) \mathbf{Q}_{2}\right) \leq 2 / 3$. This also guarantees that $\mathcal{Q}_{B}$ is closed and bounded. Then we introduce the set of uniaxial (Ericksen) tensors, namely, the one obtained when the de Gennes order tensors are constrained to be uniaxial, i.e., when two eigenvalues coincide,

$$
\begin{aligned}
\mathcal{Q}_{U}:= & \left\{\mathbf{Q} \in \mathbb{M}_{\text {sym }}^{3 \times 3}: \operatorname{tr} \mathbf{Q}=0,-\frac{1}{3} \leq \lambda_{\min }(\mathbf{Q}) \leq \lambda_{\max }(\mathbf{Q}) \leq \frac{2}{3}\right. \\
& \left.\lambda_{\max }(\mathbf{Q})=-2 \lambda_{\min }(\mathbf{Q}) \quad \text { or } \quad \lambda_{\min }(\mathbf{Q})=-2 \lambda_{\max }(\mathbf{Q})\right\} .
\end{aligned}
$$

The last case is that of the Frank model, namely, the one using as nematic state variable only the eigenframe of $\mathbf{Q}$, which is constrained to have eigenvalues $2 / 3,-1 / 3,-1 / 3$ and which ranges in the set

$$
\mathcal{Q}_{F r}:=\left\{\mathbf{Q} \in \mathbb{M}_{\text {sym }}^{3 \times 3}: \operatorname{tr} \mathbf{Q}=0, \lambda_{\max }(\mathbf{Q})=\frac{2}{3}, \lambda_{\min }(\mathbf{Q})=-\frac{1}{3}\right\} .
$$

Notice that since $\operatorname{tr} \mathbf{Q}=0$, this suffices to describe the spectrum of $\mathbf{Q}$. It follows by definition that $\mathcal{Q}_{F r} \subset \mathcal{Q}_{U} \subset \mathcal{Q}_{B}$ and, importantly, $\mathcal{Q}_{U}$ and $\mathcal{Q}_{F r}$ are closed and nonconvex sets. Moreover, $\mathcal{Q}_{B}$ coincides with the convex envelope of $\mathcal{Q}_{F r}$ and $\mathcal{Q}_{U}[7$, Remarks 3 and 4]. 
1.1.1. Functional setting. Let $\Omega$ be an open and bounded subset of $\mathbb{R}^{n}$. We adopt the notation $C^{k}(\Omega), C^{k}\left(\Omega, \mathbb{R}^{n}\right), C^{k}\left(\Omega, \mathbb{M}^{n \times n}\right)$, with $k \in \mathbb{N} \cup\{0,+\infty\}$, for the spaces of functions with continuous derivatives up to order $k$ and $C_{c}^{k}(\Omega), C_{c}^{k}\left(\Omega, \mathbb{R}^{n}\right)$, $C_{c}^{k}\left(\Omega, \mathbb{M}^{n \times n}\right)$ for the spaces of continuous functions with compact support in $\Omega$. Letting $p \in[1,+\infty)$, we introduce $L^{p}(\Omega)$, the space of measurable functions $u: \Omega \mapsto \mathbb{R}$ such that $\int_{\Omega}|u|^{p} d x<+\infty$, and moreover $L^{p}\left(\Omega, \mathbb{R}^{n}\right)$ and $L^{p}\left(\Omega, \mathbb{M}^{n \times n}\right)$, respectively, the spaces of vectors or matrices with components in $L^{p}(\Omega)$. The space $L^{\infty}\left(\Omega, \mathbb{M}^{n \times n}\right)$ is that of matrices with essentially bounded components. Then $H^{1, p}(\Omega)$ is the space of scalar-valued $L^{p}$-functions whose gradient is in $L^{p}\left(\Omega, \mathbb{R}^{n}\right)$, and $H^{1, p}\left(\Omega, \mathbb{R}^{n}\right)$, $H^{1, p}\left(\Omega, \mathbb{M}^{n \times n}\right)$ are the spaces of vector-valued and matrix-valued $L^{p}$-functions whose gradient has $L^{p}$-integrable components. They are endowed with the usual norms and, if $p=2$, we simply write $H^{1}$ instead of $H^{1,2}$. The space $H_{o}^{1}(\Omega)$ is defined as the closure of $C_{c}^{\infty}(\Omega)$ in the topology of $H^{1}(\Omega)$ (analogously for $H_{o}^{1}\left(\Omega, \mathbb{R}^{n}\right)$ and $H_{o}^{1}\left(\Omega, \mathbb{M}^{n \times n}\right)$ ). If $\Omega$ is a domain with Lipschitz boundary, we label with $H_{\Gamma_{D}}^{1}\left(\Omega, \mathbb{R}^{n}\right)$ the subspace of $H^{1}$-functions which vanish in the sense of traces (see [10, Theorem 6.1-7]) on $\Gamma_{D}$, where $\Gamma_{D}$ is a subset of $\partial \Omega$ with positive surface measure. Moreover, let $\mathcal{Z}$ be a compact set in $\mathbb{M}^{n \times n}$. We introduce the subsets

$$
\begin{aligned}
& L^{2}(\Omega, \mathcal{Z}):=\left\{\mathbf{Z} \in L^{2}\left(\Omega, \mathbb{M}^{n \times n}\right): \mathbf{Z}(x) \in \mathcal{Z} \text { a.e. in } \Omega\right\} \\
& H^{1}(\Omega, \mathcal{Z}):=\left\{\mathbf{Z} \in H^{1}\left(\Omega, \mathbb{M}^{3 \times 3}\right): \mathbf{Z}(x) \in \mathcal{Z} \text { a.e. in } \Omega\right\} .
\end{aligned}
$$

Focusing on the case $n=3, \Omega$ is the reference domain occupied by a three-dimensional body. We denote by $\left(x_{1}, x_{2}, x_{3}\right)$ the cartesian components of the point $x$ in $\Omega$. The system is described by the displacement $\mathbf{u}: \Omega \mapsto \mathbb{R}^{3}$ and the order tensor $\mathbf{Q}: \Omega \mapsto \mathcal{Q}_{X}$, where $X$ stands for either $F r$, or $U$, or $B$ (this notation will be used throughout the paper). In particular, if we replace $\mathcal{Z}$ with $\mathcal{Q}_{X}$ in (1.8) and (1.9), we obtain the subsets of order tensors $L^{2}\left(\Omega, \mathcal{Q}_{X}\right)$ and $H^{1}\left(\Omega, \mathcal{Q}_{X}\right)$. It is straightforward to prove that $L^{2}\left(\Omega, \mathcal{Q}_{F r}\right), L^{2}\left(\Omega, \mathcal{Q}_{U}\right)$, and $L^{2}\left(\Omega, \mathcal{Q}_{B}\right)$ are closed in the strong topology of $L^{2}\left(\Omega, \mathbb{M}^{3 \times 3}\right)$. To this end, we can take a strongly convergent sequence $\left\{\mathbf{Q}_{k}\right\} \subset$ $L^{2}\left(\Omega, \mathcal{Q}_{X}\right)$ and extract a subsequence $\left\{\mathbf{Q}_{k_{j}}\right\}$ which converges pointwise. Since $\lambda_{\min }$ and $\lambda_{\max }$ are continuous functions, the claim is proved. Moreover, $L^{2}\left(\Omega, \mathcal{Q}_{B}\right)$ is closed also in the sense of the weak topology by convexity (Hahn-Banach theorem [6]). On the other hand, the sets $L^{2}\left(\Omega, \mathcal{Q}_{F r}\right)$ and $L^{2}\left(\Omega, \mathcal{Q}_{U}\right)$ are not weakly closed, and one can prove (see Remark 2 in section 2 ) that $L^{2}\left(\Omega, \mathcal{Q}_{B}\right)$ coincides with the (closed) convex envelope of $L^{2}\left(\Omega, \mathcal{Q}_{F r}\right)$ (and of $L^{2}\left(\Omega, \mathcal{Q}_{U}\right)$ ).

1.1.2. Gamma-convergence and relaxation. In this article we are mainly interested in Gamma-convergence and relaxation of functionals with respect to the product of the weak topology of $L^{2}$ with the weak topology of $H^{1}$, and with respect to the product of the strong topology of $L^{2}$ with the weak topology of $H^{1}$. Here, as a paradigm, we define the Gamma-convergence and the relaxation in the weak topology of $H^{1}$. Our general references are [4], [14]. We present the sequential characterization of the Gamma-convergence (this is equivalent to the abstract topological one since all the functionals defined in this paper satisfy a uniform coercivity condition $[14$, Chapter 8]).

Definition 1. Let $\left\{\mathcal{F}_{h}\right\}$ be a sequence of functionals defined on $H^{1}\left(\Omega, \mathbb{R}^{3}\right)$. We define for any $\mathbf{u} \in H^{1}\left(\Omega, \mathbb{R}^{3}\right)$

$$
\begin{aligned}
\Gamma-\liminf _{h \rightarrow+\infty} & \mathcal{F}_{h}(\mathbf{u})=\inf \left\{\liminf _{h \rightarrow+\infty} \mathcal{F}_{h}\left(\mathbf{u}_{h}\right), \mathbf{u}_{h} \rightarrow \mathbf{u}\right\}, \\
\Gamma-\limsup _{h \rightarrow+\infty} \mathcal{F}_{h}(\mathbf{u}) & =\inf \left\{\limsup _{h \rightarrow+\infty} \mathcal{F}_{h}\left(\mathbf{u}_{h}\right), \mathbf{u}_{h} \rightarrow \mathbf{u}\right\} .
\end{aligned}
$$


If we have

$$
\Gamma-\liminf _{h \rightarrow+\infty} \mathcal{F}_{h}(\mathbf{u})=\Gamma-\limsup _{h \rightarrow+\infty} \mathcal{F}_{h}(\mathbf{u}),
$$

then the common value is said to be the $\Gamma-\lim _{h \rightarrow+\infty} \mathcal{F}_{h}(\mathbf{u})$.

Definition 2. Let $\mathcal{F}$ be a functional defined on $H^{1}\left(\Omega, \mathbb{R}^{3}\right)$. We define the relaxation of $\mathcal{F}$ with respect to the weak topology of $H^{1}\left(\Omega, \mathbb{R}^{3}\right)$ :

$$
\overline{\mathcal{F}}=\sup \left\{\mathcal{G}: \mathcal{G} \text { is } H^{1}\left(\Omega, \mathbb{R}^{3}\right) \text { weakly lower semicontinuous, } \mathcal{G} \leq \mathcal{F}\right\} .
$$

For the reader's convenience, we recall a useful property of the Gamma-convergence which will be applied in section 2 .

Proposition 1. Let $\left\{\mathcal{F}_{h}\right\}$ be an increasing sequence of functionals defined on $H^{1}\left(\Omega, \mathbb{R}^{3}\right)$, and let $\overline{\mathcal{F}}_{h}$ be the relaxation of $\mathcal{F}_{h}$. Then for every $\mathbf{u} \in H^{1}\left(\Omega, \mathbb{R}^{3}\right)$ there exists

$$
\Gamma-\lim _{h \rightarrow+\infty} \mathcal{F}_{h}(\mathbf{u})=\Gamma-\lim _{h \rightarrow+\infty} \overline{\mathcal{F}}_{h}(\mathbf{u})=\sup _{h} \overline{\mathcal{F}}_{h}(\mathbf{u}) .
$$

1.1.3. Gathering of technical propositions. We present some preliminary results which are applied in the rest of the paper.

Korn's inequalities [10, Theorems 6.3-3 and 6.3-4]. Let $\Omega$ be an open, bounded, connected subset of $\mathbb{R}^{3}$ with Lipschitz boundary. Let $\mathbf{z} \in H^{1}\left(\Omega, \mathbb{R}^{3}\right)$. Then there exists a positive constant $K_{1}=K_{1}(\Omega)$ such that

$$
\|\nabla \mathbf{z}\|_{L^{2}\left(\Omega, \mathbb{M}^{3 \times 3}\right)}^{2} \leq K_{1}(\Omega)\left(\|\mathbf{z}\|_{L^{2}\left(\Omega, \mathbb{R}^{3}\right)}^{2}+\|\mathbb{E}(\nabla \mathbf{z})\|_{L^{2}\left(\Omega, \mathbb{M}^{3 \times 3}\right)}^{2}\right) .
$$

Now let $\mathbf{z} \in H_{\Gamma_{D}}^{1}\left(\Omega, \mathbb{R}^{3}\right)$. Then there exists a positive constant $K_{2}=K_{2}(\Omega)$ such that

$$
\|\nabla \mathbf{z}\|_{L^{2}\left(\Omega, \mathbb{M}^{3 \times 3}\right)}^{2} \leq K_{2}(\Omega)\left(\|\mathbb{E}(\nabla \mathbf{z})\|_{L^{2}\left(\Omega, \mathbb{M}^{3 \times 3}\right)}^{2}\right) .
$$

The following proposition, which is due to Bogovskiı (see [24, Theorem 3.1]), has an important role in order to treat the case of incompressible elastomers.

Proposition 2. Consider $\mathbb{N} \ni n \geq 2$ and $p \in(1, \infty)$. Let $\Omega$ be an open, bounded, connected subset of $\mathbb{R}^{n}$ with Lipschitz boundary. Assume $\mathbf{z} \in H_{o}^{1, p}\left(\Omega, \mathbb{R}^{n}\right)$. Then there exists at least one solution to the problem

$$
\left\{\begin{array}{c}
\mathbf{w} \in H_{o}^{1, p}\left(\Omega, \mathbb{R}^{n}\right), \\
\operatorname{div} \mathbf{w}=\operatorname{div} \mathbf{z} \\
\|\mathbf{w}\|_{H^{1, p}\left(\Omega, \mathbb{R}^{n}\right)} \leq C(\Omega, n, p)\|\operatorname{div} \mathbf{z}\|_{L^{p}(\Omega)} .
\end{array}\right.
$$

In the rest of the paragraph, let $\Omega \subset \mathbb{R}^{d}$ with $\mathbb{N} \ni d \geq 2$ be a (nonempty) open, bounded, and connected set with Lipschitz boundary, and let $\mathcal{K} \subset \mathbb{M}^{d \times d}$ be a compact and convex set.

Proposition 3. Let $\mathbf{Q} \in L^{2}(\Omega, \mathcal{K})$. Then there exists a sequence $\left\{\mathbf{Q}_{k}\right\} \subset$ $L^{2}(\Omega, \mathcal{K})$ of piecewise-constant matrices such that $\mathbf{Q}_{k} \rightarrow \mathbf{Q}$ strongly in $L^{2}\left(\Omega, \mathbb{M}^{d \times d}\right)$ as $k \rightarrow+\infty$.

Proof. We recall that a function $\mathbf{Q}$ is said to be piecewise-constant if there exists a partition of $\Omega$ consisting of a finite number $m$ of open and pairwise disjoint sets $\Omega^{j}$ such that

$$
\Omega=\bigcup_{j=1}^{m} \Omega^{j} \cup N
$$

Copyright $@$ by SIAM. Unauthorized reproduction of this article is prohibited. 
where $|N|=0$ and $\mathbf{Q}^{j}:=\left.\mathbf{Q}\right|_{\Omega^{j}}$ is constant. We can take a sequence $\left\{\mathbf{Z}_{k}\right\} \subset$ $L^{2}\left(\Omega, \mathbb{M}^{d \times d}\right)$ of piecewise-constant matrices such that $\mathbf{Z}_{k} \rightarrow \mathbf{Q}$ strongly in $L^{2}\left(\Omega, \mathbb{M}^{d \times d}\right)$ (this is a standard approximation result; see, for instance, the construction in the proof of [16, Proposition 2.2] and the references cited therein). Then, if we define $\mathbf{Q}_{k}: \pi^{\mathcal{K}}\left(\mathbf{Z}_{k}\right)$ for any $k$, we obtain the new sequence $\left\{\mathbf{Q}_{k}\right\} \subset L^{2}(\Omega, \mathcal{K})$ of piecewiseconstant tensors, which proves the claim.

In what follows we assume $d=3$.

Lemma 1. For any $\mathbf{Q} \in L^{2}\left(\Omega, \mathcal{Q}_{F r}\right)$ there exists $\left\{\mathbf{Q}_{k}\right\} \subset H^{1}\left(\Omega, \mathcal{Q}_{F r}\right)$ such that

$$
\mathbf{Q}_{k} \rightarrow \mathbf{Q} \text { in } L^{2}\left(\Omega, \mathbb{M}^{3 \times 3}\right) \text { as } k \rightarrow+\infty .
$$

Proof. It is easy to show that any $\mathbf{Q} \in L^{2}\left(\Omega, \mathcal{Q}_{F r}\right)$ can be written in the form $\mathbf{Q}=\mathbf{n} \otimes \mathbf{n}-\frac{1}{3} \mathbf{I}$ with $\mathbf{n} \in L^{2}\left(\Omega, \mathbb{S}^{2}\right)$. By the spectral theorem it is possible to set $\mathbf{Q}$ in diagonal form

$$
\mathbf{Q}=\frac{2}{3} \mathbf{n} \otimes \mathbf{n}-\frac{1}{3} \mathbf{m} \otimes \mathbf{m}-\frac{1}{3} \mathbf{p} \otimes \mathbf{p},
$$

where $\{\mathbf{n}, \mathbf{m}, \mathbf{p}\}$ is an orthonormal frame. Since two of the eigenvalues of $\mathbf{Q}$ coincide, (1.14) is equivalent to

$$
\mathbf{Q}=\frac{2}{3} \mathbf{n} \otimes \mathbf{n}-\frac{1}{3}(\mathbf{I}-\mathbf{n} \otimes \mathbf{n})=\left(\mathbf{n} \otimes \mathbf{n}-\frac{1}{3} \mathbf{I}\right) .
$$

Now, for any given $\mathbf{n} \in L^{2}\left(\Omega, \mathbb{S}^{2}\right)$, there exists a sequence

$$
\mathbf{n}_{k} \rightarrow \mathbf{n} \text { in } L^{2}\left(\Omega, \mathbb{R}^{3}\right) \quad \text { as } \quad k \rightarrow+\infty
$$

with $\left\{\mathbf{n}_{k}\right\} \subset H^{1}\left(\Omega, \mathbb{S}^{2}\right)$ (see [16, Assertion 1, pages 109-110, with $\left.m_{s}=1\right]$ ). Let us define

$$
\mathbf{Q}_{k}:=\mathbf{n}_{k} \otimes \mathbf{n}_{k}-\frac{1}{3} \mathbf{I}
$$

Every tensor field $\mathbf{Q}_{k}$ belongs to $H^{1}\left(\Omega, \mathcal{Q}_{F r}\right)$ by an elementary property of the product of essentially bounded $H^{1}$-functions. Then it is sufficient to verify that $\mathbf{n}_{k} \otimes \mathbf{n}_{k} \rightarrow \mathbf{n} \otimes \mathbf{n}$ in $L^{2}\left(\Omega, \mathbb{M}^{3 \times 3}\right)$ :

$$
\begin{aligned}
\int_{\Omega}\left|\mathbf{n}_{k} \otimes \mathbf{n}_{k}-\mathbf{n} \otimes \mathbf{n}\right|^{2} d x=\int_{\Omega}\left|\mathbf{n}_{k} \otimes \mathbf{n}_{k}-\mathbf{n}_{k} \otimes \mathbf{n}+\mathbf{n}_{k} \otimes \mathbf{n}-\mathbf{n} \otimes \mathbf{n}\right|^{2} d x \\
\leq a_{1} \int_{\Omega}\left|\mathbf{n}_{k}\right|\left|\mathbf{n}_{k}-\mathbf{n}\right|^{2} d x+a_{2} \int_{\Omega}|\mathbf{n}|\left|\mathbf{n}_{k}-\mathbf{n}\right|^{2} d x \stackrel{k \rightarrow \infty}{\longrightarrow} 0,
\end{aligned}
$$

where $a_{1}, a_{2}$ are suitable positive constants.

Remark 1 . The lemma above proves that any Frank tensor in $L^{2}\left(\Omega, \mathcal{Q}_{F r}\right)$ can be approximated in the strong topology by a sequence of oriented Frank tensors in the sense of $[2]$.

Properties of the distance. Given any matrix $\mathbf{F} \in \mathbb{M}^{3 \times 3}$ and a positive number $\gamma$, we introduce $\overline{\mathbf{Q}}:=\pi^{\mathcal{Q}_{B}}(\mathbb{E}(\mathbf{F}) / \gamma)=\frac{1}{\gamma} \pi^{\gamma \mathcal{Q}_{B}}(\mathbb{E}(\mathbf{F}))$, the projection of the symmetric part of $\mathbf{F} / \gamma$ onto the subset $\mathcal{Q}_{B}$. By definition $\overline{\mathbf{Q}}$ is the element of minimum distance from the set $\mathcal{Q}_{B}$, and it follows that

$$
\operatorname{dist}^{2}\left(\mathbb{E}(\mathbf{F}), \gamma \mathcal{Q}_{B}\right)=\min _{\mathbf{Q} \in \mathcal{Q}_{B}}|\mathbb{E}(\mathbf{F})-\gamma \mathbf{Q}|^{2}=|\mathbb{E}(\mathbf{F})-\gamma \overline{\mathbf{Q}}|^{2} .
$$

Copyright $@$ by SIAM. Unauthorized reproduction of this article is prohibited. 
The projection is defined uniquely by the convexity and compactness of $\mathcal{Q}_{B}$. Moreover, the map $\mathbf{F} \mapsto \pi^{\gamma \mathcal{Q}_{B}}(\mathbb{E}(\mathbf{F}))$ is Lipschitz-continuous in the space of matrices.

With some abuse of notation, given any tensor field $\mathbf{F} \in L^{2}\left(\Omega, \mathbb{M}^{3 \times 3}\right)$, we define with $\overline{\mathbf{Q}}(x):=\pi^{L^{2}\left(\Omega, \mathcal{Q}_{B}\right)}(\mathbb{E}(\mathbf{F}) / \gamma)(x)=\frac{1}{\gamma} \pi^{L^{2}\left(\Omega, \gamma \mathcal{Q}_{B}\right)}(\mathbb{E}(\mathbf{F}))(x)$ the projection of the symmetric part of $\mathbf{F} / \gamma$ onto $L^{2}\left(\Omega, \mathcal{Q}_{B}\right)$. Again, the projection is defined uniquely because $L^{2}\left(\Omega, \mathcal{Q}_{B}\right)$ is a convex, closed, and bounded subset of $L^{2}\left(\Omega, \mathbb{M}^{3 \times 3}\right)$, and it follows that $\mathbf{F} \mapsto \pi^{L^{2}\left(\Omega, \gamma \mathcal{Q}_{B}\right)}(\mathbb{E}(\mathbf{F}))$ is a Lipschitz-continuous map in the $L^{2}\left(\Omega, \mathbb{M}^{3 \times 3}\right)$ metric. We have

$$
\begin{aligned}
\operatorname{dist}_{L^{2}}^{2}\left(\mathbb{E}(\mathbf{F}), L^{2}\left(\Omega, \gamma \mathcal{Q}_{B}\right)\right) & =\min _{\mathbf{Q} \in L^{2}\left(\Omega, \mathcal{Q}_{B}\right)}\|\mathbb{E}(\mathbf{F})-\gamma \mathbf{Q}\|_{L^{2}\left(\Omega, \mathbb{M}^{3 \times 3}\right)}^{2} \\
& =\int_{\Omega}|\mathbb{E}(\mathbf{F})(x)-\gamma \overline{\mathbf{Q}}(x)|^{2} d x .
\end{aligned}
$$

The relation between $\pi^{\gamma \mathcal{Q}_{B}}(\cdot)$ and $\pi^{L^{2}\left(\Omega, \gamma \mathcal{Q}_{B}\right)}(\cdot)$ is shown in the next elementary proposition, which we do not prove.

Proposition 4. Given any $\mathbf{F} \in L^{2}\left(\Omega, \mathbb{M}^{3 \times 3}\right)$, then

$$
\left(\pi^{L^{2}\left(\Omega, \gamma \mathcal{Q}_{B}\right)}(\mathbf{F})\right)(x)=\pi^{\gamma \mathcal{Q}_{B}}(\mathbf{F}(x))
$$

for a.e. $x \in \Omega$.

A consequence of Proposition 4 is that

$$
\operatorname{dist}_{L^{2}}^{2}\left(\mathbb{E}(\mathbf{F}), L^{2}\left(\Omega, \gamma \mathcal{Q}_{B}\right)\right)=\int_{\Omega} \operatorname{dist}^{2}\left(\mathbb{E}(\mathbf{F}(x)), \gamma \mathcal{Q}_{B}\right) d x .
$$

1.2. The mechanical model. According to [8], we describe the equilibrium configurations of a system of nematic elastomers as the minimizers of the energy functional

$$
\mathcal{F}^{\kappa}(\mathbf{Q}, \mathbf{u} ; \mathcal{B}):=\int_{\mathcal{B}}\left(\frac{\kappa^{2}}{2}|\nabla \mathbf{Q}|^{2}+f_{m e c}(\mathbf{Q}, \nabla \mathbf{u})\right) d x
$$

where $\mathcal{B}$ is the region occupied by the material (an open, bounded, and connected set in $\mathbb{R}^{3}$ with Lipschitz boundary). Each of the summands in (1.18) can be regarded as a penalty term that enforces a certain feature of the observed behavior of nematic elastomers. The term $\frac{\kappa^{2}}{2}|\nabla \mathbf{Q}|^{2}$ is typical in the literature on liquid crystals and is introduced in order to penalize spatial variations of $\mathbf{Q}$. The material parameter $\kappa^{2}$ is called the curvature constant, and the corresponding energy contribution is called the curvature energy. Expressions for the curvature energy more general than that used here are possible. All the results of this paper are still valid if we replace $\frac{\kappa^{2}}{2}|\nabla \mathbf{Q}|^{2}$ with the more general expression

$$
\frac{L_{1}}{2} Q_{\alpha \beta, \gamma} Q_{\alpha \beta, \gamma}+\frac{L_{2}}{2} Q_{\alpha \beta, \beta} Q_{\alpha \gamma, \gamma} \frac{L_{3}}{2} Q_{\alpha \beta, \gamma} Q_{\alpha \gamma, \beta},
$$

where $L_{i}, i=1,2,3$, are the elastic constants and $Q_{\alpha \beta, \gamma}$ is the $\alpha \beta$ Cartesian component of the first derivative of $\mathbf{Q}$ with respect to $x_{\gamma}(\alpha, \beta, \gamma=1,2,3)$. Summation over repeated indices is understood. The constants $L_{i}$ satisfy some constraints (see [26]) in order to make (1.19) a positive definite expression of the first-order derivatives of $\mathbf{Q}$, and the choice $L_{1}=\kappa^{2}, L_{2}=0, L_{3}=0$ well satisfies the constraints. The mechanical 
energy density $f_{m e c}$ is a positive function which describes the coupling between strain and order in nematic elastomers in the form

$$
\begin{array}{r}
(1.20) f_{\text {mec }}(\mathbf{Q}, \mathbf{F}):=\frac{1}{2} \mathbb{C}(\mathbb{E}(\mathbf{F})-\gamma \mathbf{Q}):(\mathbb{E}(\mathbf{F})-\gamma \mathbf{Q})=\mu|\mathbb{E}(\mathbf{F})-\gamma \mathbf{Q}|^{2}+\frac{\lambda}{2}(\operatorname{tr} \mathbf{F})^{2}, \\
\mathbf{Q} \in \mathcal{Q}_{X}, \mathbf{F} \in \mathbb{M}^{3 \times 3} \text {, where } X \text { stands for either } F r \text { or } U \text {, or } B .
\end{array}
$$

Here $\mu$ and $\lambda$ are the positive Lamé constants of linearized isotropic elasticity, and $\gamma$ is another positive and dimensionless constant. Accepting (1.20) has nontrivial physical consequences. In view of (1.20), the mechanical energy vanishes if and only if the order tensor can reproduce the mechanical strain $\left(\mathbf{Q}=\frac{1}{\gamma} \mathbb{E}(\mathbf{F})\right)$, and when $\mathbf{Q}$ differs from $\frac{1}{\gamma} \mathbb{E}(\mathbf{F})$, there is a finite penalization for any value of $\mathbf{Q}$ within the admissible set $\mathcal{Q}_{X}$. In particular, in Frank's scenario the main effect of the coupling between liquid crystalline order and elasticity is the possibility of reorienting the molecules through applied forces or imposed displacements, while in the more general scenario of de Gennes and Ericksen, also the degree of order may be affected by mechanical means (see also [11], [12], [13], [17], [18], [19]). Since $\mathcal{Q}_{B}$ is compact, there exist $C_{1}, C_{2}$, and $C_{3}$ positive constants such that

$$
(1.21)-C_{1}+C_{2}|\mathbb{E}(\mathbf{F})|^{2} \leq f_{m e c}(\mathbf{Q}, \mathbf{F}) \leq C_{3}\left(1+|\mathbb{E}(\mathbf{F})|^{2}\right) \quad \forall \mathbf{Q} \in \mathcal{Q}_{X}, \mathbf{F} \in \mathbb{M}^{3 \times 3},
$$

where $X$ stands for either $F r$, or $U$, or $B$. Furthermore, experimental observations show that nematic elastomers are nearly incompressible (the bulk modulus is orders of magnitude larger than the shear modulus). The classical way to model such materials in linearized elasticity is to restrict the admissible deformation gradients to the class of traceless matrices or, equivalently, to introduce a constraint on the divergence of the displacement.

The investigation of the equilibrium configurations of a system of nematic elastomers, namely, the solution to the problem

(1.22) minimize $\mathcal{F}^{\kappa}(\mathbf{Q}, \mathbf{u} ; \mathcal{B})$, with $\mathbf{Q} \in H^{1}\left(\mathcal{B}, \mathcal{Q}_{X}\right)$ and $\mathbf{u} \in H_{o}^{1}\left(\mathcal{B}, \mathbb{R}^{3}\right), \operatorname{div} \mathbf{u}=0$,

where $\kappa^{2}$ is an assigned (and nonzero) constant and $X$ stands for either $F r$, or $U$, or $B$, is the subject of [8, Theorem 2.2]. Briefly, existence of minimizers is guaranteed by the boundedness of minimizing sequences and the weak sequential lower semicontinuity of $(\mathbf{Q}, \mathbf{u}) \rightarrow \mathcal{F}^{\kappa}(\cdot, \cdot ; \mathcal{B})$ with respect to the product of the weak topology of $H^{1}\left(\mathcal{B}, \mathbb{M}^{3 \times 3}\right)$ with the weak topology of $H^{1}\left(\mathcal{B}, \mathbb{R}^{3}\right)$. It is important to note that, in [8], all the material and the physical parameters of the system are assumed to be constant.

According to experimental observations, in very small specimens of a nematic gel the nematic molecules tend to be uniformly aligned to some common direction, while in sufficiently large samples they can form fine domain structures. To understand how the energy terms in (1.18) are affected by a volume rescaling, consider a domain $\mathcal{B} \subset$ $\mathbb{R}^{3}$ of volume $\Lambda^{3}$, where $\Lambda \in(0, \infty)$ and let $\mathbf{Q}_{\Lambda} \in H^{1}\left(\mathcal{B}, \mathcal{Q}_{F r}\right)$ and $\mathbf{u}_{\Lambda} \in H_{o}^{1}\left(\mathcal{B}, \mathbb{R}^{3}\right)$. Set $\Omega:=(1 / \Lambda) \mathcal{B}$ and define $\mathbf{Q}: \Omega \mapsto \mathcal{Q}_{X}, \mathbf{u}: \Omega \mapsto \mathbb{R}^{3}$ by

$$
\mathbf{Q}\left(\frac{1}{\Lambda} z\right):=\mathbf{Q}_{\Lambda}(z), \quad \Lambda \mathbf{u}\left(\frac{1}{\Lambda} z\right):=\mathbf{u}_{\Lambda}(z), \quad z \in \mathcal{B} .
$$

Obviously, $\Omega$ is an open, bounded, connected subset of $\mathbb{R}^{3}$ with Lipschitz boundary and unit volume, $\mathbf{Q} \in H^{1}\left(\Omega, \mathcal{Q}_{X}\right)$, and $\mathbf{u} \in H_{o}^{1}\left(\Omega, \mathbb{R}^{3}\right)$. Hence, it follows that

$(1.23) \frac{1}{|\mathcal{B}|} \mathcal{F}^{\kappa}\left(\mathbf{Q}_{\Lambda}, \mathbf{u}_{\Lambda} ; \mathcal{B}\right)=\mathcal{F}^{\kappa / \Lambda}(\mathbf{Q}, \mathbf{u} ; \Omega)=\int_{\Omega}\left(\frac{\kappa^{2}}{2 \Lambda^{2}}|\nabla \mathbf{Q}|^{2}+f_{m e c}(\mathbf{Q}, \nabla \mathbf{u})\right) d x$, 
and, if we set $\varepsilon^{2}:=\kappa^{2} / 2 \Lambda^{2}$, we obtain the integral expression presented in the introduction. In what follows, we analyze the effect of the variation of the size of the domain by studying the minimizers for the reference configuration $\Omega$ in the two relevant limits as the scale parameter $\Lambda$ approaches the ends of its range by means of Gamma-convergence.

2. Large bodies: Asymptotics. We discuss the asymptotic behavior of large samples of nematic elastomers by computing the Gamma-limit of the functional

$$
\mathcal{F}_{X, \varepsilon}(\mathbf{Q}, \mathbf{u}):=\left\{\begin{array}{r}
\int_{\Omega}\left(\varepsilon^{2}|\nabla \mathbf{Q}|^{2}+f_{\text {mec }}(\mathbf{Q}, \nabla \mathbf{u})\right) d x \\
+\infty \quad \begin{array}{l}
\text { on } H^{1}\left(\Omega, \mathcal{Q}_{X}\right) \times H^{1}\left(\Omega, \mathbb{R}^{3}\right), \operatorname{div} \mathbf{u}=0, \\
\text { otherwise in } L^{2}\left(\Omega, \mathbb{M}^{3 \times 3}\right) \times H^{1}\left(\Omega, \mathbb{R}^{3}\right),
\end{array}
\end{array}\right.
$$

where $X$ stands for either $F r$, or $U$, or $B$ as $\varepsilon \rightarrow 0$ in the topology

$$
\sigma:=\text { weak- } L^{2}\left(\Omega, \mathbb{M}^{3 \times 3}\right) \times \text { weak- } H^{1}\left(\Omega, \mathbb{R}^{3}\right) .
$$

For convenience, we adopt the shortened notation

$$
\mathbf{Q}_{k}, \mathbf{u}_{k} \stackrel{\sigma}{\rightarrow} \mathbf{Q}, \mathbf{u} \quad \text { as } k \rightarrow+\infty
$$

which stands for

$$
\mathbf{u}_{k} \rightarrow \mathbf{u} \text { in } H^{1}\left(\Omega, \mathbb{R}^{3}\right), \quad \mathbf{Q}_{k} \rightarrow \mathbf{Q} \text { in } L^{2}\left(\Omega, \mathbb{M}^{3 \times 3}\right) \text { as } k \rightarrow+\infty .
$$

We recall that the Gamma-convergence in the weak topology of $H^{1}\left(\Omega, \mathbb{R}^{3}\right)$ is equivalent to the Gamma-convergence in the strong topology of $L^{2}\left(\Omega, \mathbb{R}^{3}\right)$ because the functional bounds the $L^{2}$-norm of the gradient of $\mathbf{u}$ by (1.21) and Korn's inequality (1.11). Analogously, since $L^{2}\left(\Omega, \mathcal{Q}_{B}\right)$ is contained in a closed and bounded ball of $L^{2}\left(\Omega, \mathbb{M}^{3 \times 3}\right)$, then the weak topology over $L^{2}\left(\Omega, \mathcal{Q}_{X}\right)$ is metrizable [14, Chapter 8]. If we define

$$
\mathcal{F}_{X, m e c}(\mathbf{Q}, \mathbf{u}):=\left\{\begin{array}{cc}
\int_{\Omega} f_{m e c}(\mathbf{Q}, \nabla \mathbf{u}) d x & \text { on } L^{2}\left(\Omega, \mathcal{Q}_{X}\right) \times H^{1}\left(\Omega, \mathbb{R}^{3}\right), \operatorname{div} \mathbf{u}=0 \\
+\infty & \text { otherwise in } L^{2}\left(\Omega, \mathbb{M}^{3 \times 3}\right) \times H^{1}\left(\Omega, \mathbb{R}^{3}\right),
\end{array}\right.
$$

where $X$ stands for either $F r$, or $U$, or $B$, we claim that $\mathcal{F}_{X, \varepsilon}$ Gamma-converges to the relaxation of $\mathcal{F}_{X, m e c}$. Therefore, it is natural to split the proof into two main parts, the first one (section 2.1) in which we compute the relaxation of $\mathcal{F}_{X, m e c}$, and the second part (section 2.2) containing the Gamma-convergence theorem.

2.1. Relaxation. According to well-known techniques in the calculus of variations [14], [22], in order to relax $\mathcal{F}_{X, \text { mec }}$ we can exhibit a suitable recovery sequence for any $(\mathbf{Q}, \mathbf{u}) \in L^{2}\left(\Omega, \mathbb{M}^{3 \times 3}\right) \times H^{1}\left(\Omega, \mathbb{R}^{3}\right)$. Unfortunately, the presence of constraints on the components of the order tensor $\mathbf{Q} \in \mathcal{Q}_{X}$ and, in particular, on the divergence of $\mathbf{u}$, makes the construction of such a sequence a nontrivial problem. In our particular case, however, an argument due to Braides [5] and largely employed also in [7] is sufficient to compute the relaxation of $\mathcal{F}_{X, \text { mec }}$. Indeed, it is possible to show that the relaxation of the incompressible models coincides with the Gamma-limit of a sequence of relaxed energies for compressible elastomers as the bulk modulus tends to $+\infty$. According to this technique, in what follows, we introduce the functionals for compressible elastomers and compute their relaxation (Theorem 1). In this case we do not have to take into account any additional constraint on the divergence of $\mathbf{u}$, and an explicit construction of the recovery sequence turns out to be possible. Then we apply the result for compressible elastomers in order to compute the relaxation of $\mathcal{F}_{X, \text { mec }}($ Theorem 2). 


\subsubsection{The case of compressible elastomers.}

THEOREM 1. Let $\Omega \subset \mathbb{R}^{3}$ be an open, bounded, and connected set with Lipschitz boundary. Assume $f_{m e c}$ as in (1.20) and define

$$
\mathrm{F}_{X, m e c}(\mathbf{Q}, \mathbf{u}):=\left\{\begin{array}{rr}
\int_{\Omega} f_{m e c}(\mathbf{Q}, \nabla \mathbf{u}) d x \quad \text { on } L^{2}\left(\Omega, \mathcal{Q}_{X}\right) \times H^{1}\left(\Omega, \mathbb{R}^{3}\right), \\
+\infty & \text { otherwise in } L^{2}\left(\Omega, \mathbb{M}^{3 \times 3}\right) \times H^{1}\left(\Omega, \mathbb{R}^{3}\right)
\end{array}\right.
$$

where $X$ stands for either $F r$, or $U$, or $B$. Then the relaxation of $\mathrm{F}_{X, m e c}$ in the sense of $\sigma$ (see (2.1)) is $\mathrm{F}_{B, \text { mec }}$.

Proof. In the case in which we set $X=B$ in (2.4) there is nothing to prove because $\mathrm{F}_{B \text {,mec }}$ is weakly lower semicontinuous by convexity. In the case $X=U$ the result follows immediately by an abstract argument which we postpone to section 2.2.1. Here we show the complete construction for the case $X=F r$. Recalling that $L^{2}\left(\Omega, \mathcal{Q}_{F r}\right) \subset L^{2}\left(\Omega, \mathcal{Q}_{B}\right)$, we have $\mathrm{F}_{F r, m e c} \geq \mathrm{F}_{B, m e c}$ and, by relaxing both sides, $\overline{\mathrm{F}}_{F r, m e c} \geq \mathrm{F}_{B, m e c}$. Then observe that the converse inequality

$$
\overline{\mathrm{F}}_{F r, m e c} \leq \mathrm{F}_{B, m e c}
$$

is trivial if $\mathbf{Q} \notin L^{2}\left(\Omega, \mathcal{Q}_{B}\right)$. Hence, in what follows we can prove (2.5) under the assumption that $(\mathbf{Q}, \mathbf{u}) \in L^{2}\left(\Omega, \mathcal{Q}_{B}\right) \times H^{1}\left(\Omega, \mathbb{R}^{3}\right)$ by splitting the proof into three steps. In Step 1, we discuss the relaxation in the case in which the tensor field $\mathbf{Q}$ is constant by constructing a recovery sequence $\left\{\mathbf{Q}_{n}, \mathbf{u}_{n}\right\}$. In Step 2, we prove that (2.5) holds in the case of piecewise-constant biaxial tensor fields. Lastly, in Step 3, we extend the proof of Step 2 to any $L^{2}$-biaxial tensor field.

Step $1, \mathbf{Q}(\boldsymbol{x})$ constant. Assume $\mathbf{u} \in H^{1}\left(\Omega, \mathbb{R}^{3}\right)$ and $\mathbf{Q}: \Omega \mapsto \mathcal{Q}_{B}$ and constant. For every open set $U \subset \Omega$, we provide a sequence $\left\{\mathbf{Q}_{n}, \mathbf{u}_{n}\right\} \subset L^{2}\left(\Omega, \mathcal{Q}_{F r}\right) \times H^{1}\left(\Omega, \mathbb{R}^{3}\right)$ such that

$$
\mathbf{Q}_{n} \rightarrow \mathbf{Q} \text { in } L^{2}\left(U, \mathbb{M}^{3 \times 3}\right), \quad \mathbf{u}_{n} \rightarrow \mathbf{u} \text { in } H^{1}\left(U, \mathbb{R}^{3}\right) \text { as } n \rightarrow+\infty,
$$

with $\operatorname{support}\left(\mathbf{u}_{n}-\mathbf{u}\right) \subset \subset U$ and such that

$$
\limsup _{n \rightarrow+\infty} \int_{U} f_{m e c}\left(\mathbf{Q}_{n}, \nabla \mathbf{u}_{n}\right) d x \leq \int_{U} f_{m e c}(\mathbf{Q}, \nabla \mathbf{u}) d x+o(1) .
$$

Denoting by $\chi_{U}(x)$ the function which is identically equal to 1 in $U$ and identically equal to 0 in its complementary, we can write $\mathbf{Q}(x)=\mathrm{Q} \chi_{U}(x)$ with $\mathrm{Q} \in \mathcal{Q}_{B}$. With some abuse of notation, in what follows, we do not make any distinction between $\mathbf{Q}$ and $\mathrm{Q}$. By the spectral theorem there exists a rotation $\mathbf{R} \in \mathbb{S O}(3)$ such that the diagonal form of $\mathbf{Q}$ (denoted by $\mathbf{Q}_{D}$ ) reads

$$
\mathbf{Q}_{D}=\mathbf{R}^{T} \mathbf{Q R}=\left(\begin{array}{ccc}
a & 0 & 0 \\
0 & b & 0 \\
0 & 0 & c
\end{array}\right)
$$

with $a \leq b \leq c, a+b+c=0$. We can now start the construction of a weakly convergent sequence of matrices $\left\{\mathbf{F}_{n}\right\}$ whose symmetric part yields the sequence $\left\{\mathbf{Q}_{n}\right\}$ and which is itself strictly related to the sequence of gradients $\left\{\nabla \mathbf{u}_{n}\right\}$. To this end, we need to define a tensor field $\mathbf{H}$ over a bounded set and to extend it by periodicity in $\mathbb{R}^{3}$ (we 

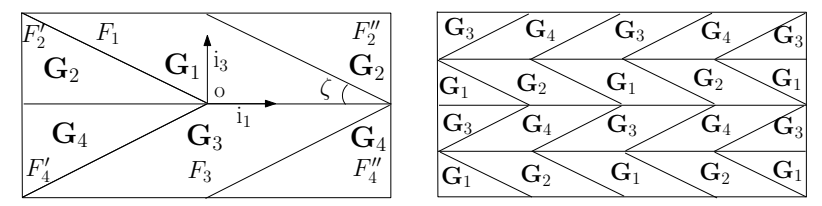

FIG. 1. Geometry of the oscillating sequence $\mathbf{F}_{n}$ in the case $a \neq-1 / 3$. The first panel depicts the case $n=1$, and the second panel the case $n=2$. The tangent of $\zeta$ has absolute value equal to $1 / T$. Here $\mathbf{R}=\mathbf{I}$.

address the interested readers to [22, section 2.2.2] as a general reference). Suppose for a moment that $a \neq-1 / 3$. Let

$$
T:=\sqrt{\frac{c+1 / 3}{a+1 / 3}}
$$

and define $\mathcal{D}:=(-T, T) \times(-1,1) \times(-1,1)$. Note that $\mathcal{D}$ can be written as the union of the subregions $F_{1}, F_{2}, F_{3}$, and $F_{4}$ (see Figure 1 ) defined below:

$$
\begin{aligned}
F_{1} & :=\left\{\left(x_{1}, x_{2}, x_{3}\right):-\frac{1}{T} x_{1} \leq x_{3}<-\frac{1}{T}\left(x_{1}-T\right), 0 \leq x_{3}<1,-T<x_{1}<T,-1<x_{2}<1\right\}, \\
F_{3} & :=\left\{\left(x_{1}, x_{2}, x_{3}\right): \frac{1}{T}\left(x_{1}-T\right)<x_{3} \leq \frac{1}{T} x_{1},-1<x_{3}<0,-T<x_{1}<T,-1<x_{2}<1\right\}, \\
F_{2}^{\prime} & :=\left\{\left(x_{1}, x_{2}, x_{3}\right): x_{3}<-\frac{1}{T} x_{1}, 0 \leq x_{3}<1,-T<x_{1} \leq 0,-1<x_{2}<1\right\}, \\
F_{2}^{\prime \prime} & :=\left\{\left(x_{1}, x_{2}, x_{3}\right):-\frac{1}{T}\left(x_{1}-T\right) \leq x_{3}, 0 \leq x_{3}<1,0<x_{1}<T,-1<x_{2}<1\right\}, \\
F_{4}^{\prime} & :=\left\{\left(x_{1}, x_{2}, x_{3}\right): x_{3}>\frac{1}{T} x_{1},-1<x_{3}<0,-T<x_{1}<0,-1<x_{2}<1\right\}, \\
F_{4}^{\prime \prime} & :=\left\{\left(x_{1}, x_{2}, x_{3}\right): x_{3} \leq \frac{1}{T}\left(x_{1}-T\right),-1<x_{3}<0,0<x_{1}<T,-1<x_{2}<1\right\}, \\
F_{2} & :=F_{2}^{\prime} \cup F_{2}^{\prime \prime}, \quad F_{4}:=F_{4}^{\prime} \cup F_{4}^{\prime \prime} .
\end{aligned}
$$

Then let $\mathbf{G}: \mathcal{D} \rightarrow \mathbb{M}^{3 \times 3}$ defined as $\mathbf{G}(x):=\mathbf{G}_{i}, i=1,2,3,4$, where $\mathbf{G}_{i}$ are the constant matrices

$$
\begin{aligned}
\mathbf{G}_{1}:=\left(\begin{array}{ccc}
a & 0 & 2 G_{a, c} \\
-2 G_{a, b} & b & -2 G_{b, c} \\
0 & 0 & c
\end{array}\right), & \mathbf{G}_{2}:=\left(\begin{array}{cccc}
a & 0 & 2 G_{a, c} \\
2 G_{a, b} & b & 2 G_{b, c} \\
0 & 0 & c
\end{array}\right), \\
\mathbf{G}_{3}:=\left(\begin{array}{ccc}
a & 0 & -2 G_{a, c} \\
-2 G_{a, b} & b & 2 G_{b, c} \\
0 & 0 & c
\end{array}\right), & \mathbf{G}_{4}:=\left(\begin{array}{ccc}
a & 0 & -2 G_{a, c} \\
2 G_{a, b} & b & -2 G_{b, c} \\
0 & 0 & c
\end{array}\right),
\end{aligned}
$$

with

$$
G_{a, b}:=\sqrt{a+\frac{1}{3}} \sqrt{b+\frac{1}{3}}, \quad G_{a, c}:=\sqrt{a+\frac{1}{3}} \sqrt{c+\frac{1}{3}}, \quad G_{b, c}:=\sqrt{b+\frac{1}{3}} \sqrt{c+\frac{1}{3}} .
$$

Suppose now that $a=-1 / 3$. In this case we denote by $\mathcal{D}$ the open cube in $\mathbb{R}^{3}$, that is, $\mathcal{D}:=(-1,1) \times(-1,1) \times(-1,1)$, and we note that $\mathcal{D}$ can be written as the union of the regions $F_{5}$ and $F_{6}$ (see Figure 2 (left)) defined below:

$$
F_{5}:=\left\{\left(x_{1}, x_{2}, x_{3}\right) \in \mathcal{D}: 0 \leq x_{3}<1\right\}, F_{6}:=\left\{\left(x_{1}, x_{2}, x_{3}\right) \in \mathcal{D}:-1<x_{3}<0\right\} .
$$

Copyright $@$ by SIAM. Unauthorized reproduction of this article is prohibited. 

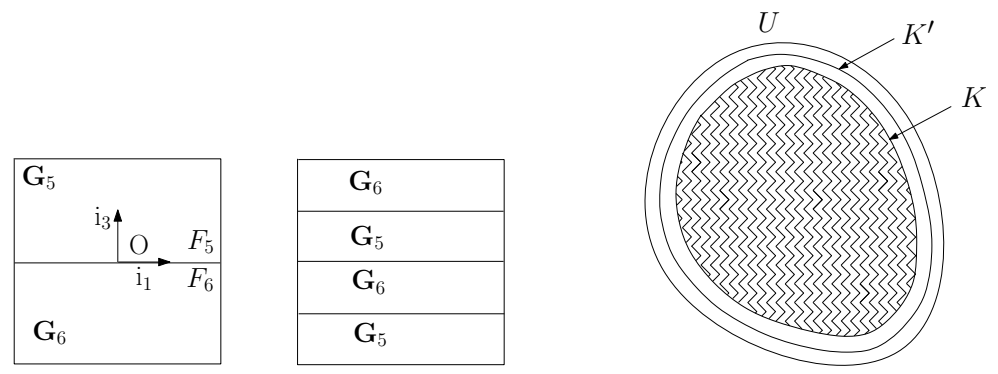

FIG. 2. Left: Geometry of the oscillating sequence $\mathbf{F}_{n}$ in the case $a=-1 / 3$. The first panel depicts the case $n=1$ and the second panel the case $n=2$. Here $\mathbf{R}=\mathbf{I}$. Right: Sketch of the construction of the sequence $\left\{\mathbf{u}_{n}\right\}$, case $a=c=0$.

Then let $\mathbf{G}: \mathcal{D} \rightarrow \mathbb{M}^{3 \times 3}$ defined as $\mathbf{G}(x):=\mathbf{G}_{i}, i=5,6$, where

$$
\mathbf{G}_{5}:=\left(\begin{array}{ccc}
-\frac{1}{3} & 0 & 0 \\
0 & b & -2 G_{b, c} \\
0 & 0 & c
\end{array}\right), \quad \mathbf{G}_{6}:=\left(\begin{array}{ccc}
-\frac{1}{3} & 0 & 0 \\
0 & b & 2 G_{b, c} \\
0 & 0 & c
\end{array}\right),
$$

and with $G_{b, c}$ defined in (2.9). Having defined $\mathbf{G}$ for any value of the parameter $a$, a straightforward computation shows that the symmetric part of $\mathbf{G}$ ranges in $\mathcal{Q}_{F r}$ since

$$
\operatorname{spectrum}\left(\mathbb{E}\left(\mathbf{G}_{i}\right)\right)=\left\{-\frac{1}{3},-\frac{1}{3}, \frac{2}{3}\right\}, \quad i=1, \ldots, 6 .
$$

Now, let $\mathbf{H}(x):=\mathbf{R} \mathbf{G} \mathbf{R}^{T}$, and let $\widetilde{\mathbf{H}}(x)$ be the extension of $\mathbf{H}(x)$ in $\mathbb{R}^{3}$ by periodicity. Define

$$
\mathbf{F}_{n}(x):=\widetilde{\mathbf{H}}\left(n x_{1}, n x_{2}, n x_{3}\right), \quad n \in \mathbb{N} .
$$

It is easy to show that

$$
\mathbf{F}_{n}(x) \stackrel{*}{\rightarrow} \mathbf{Q} \text { in } L^{\infty}\left(U, \mathbb{M}^{3 \times 3}\right) \text { as } n \rightarrow+\infty .
$$

To prove (2.13) it is sufficient to observe that the coefficients of the diagonal matrix $\mathbf{Q}_{D}$ can be obtained either as a convex combination of the matrices $\mathbf{G}_{i}$ with $i=1, \ldots, 4$ with coefficients equal to $1 / 4$ if $a \neq-1 / 3$ or as a convex combination of the matrices $\mathbf{G}_{i}$ with $i=5,6$ with coefficients equal to $1 / 2$ if $a=-1 / 3$. As anticipated, the construction presented above has a double purpose. On the one hand, by (2.12) and (2.13), we can define the new sequence $\left\{\mathbf{Q}_{n}\right\}:=\left\{\mathbb{E}\left(\mathbf{F}_{n}\right)\right\} \subset L^{2}\left(\Omega, \mathcal{Q}_{F r}\right)$ such that

$$
\mathbf{Q}_{n}(x) \stackrel{*}{\rightarrow} \mathbf{Q} \text { in } L^{\infty}\left(U, \mathbb{M}^{3 \times 3}\right) \text { as } n \rightarrow+\infty .
$$

On the other hand, we are able to construct the sequence of vectors $\left\{\mathbf{f}_{n}\right\}$ such that $\mathbf{f}_{n}=\nabla \mathbf{F}_{n}$ and yielding, with a few more changes, the desired sequence $\left\{\mathbf{u}_{n}\right\}$. To this end, we define the vector $\mathbf{g}: \mathcal{D} \rightarrow \mathbb{R}^{3}$ such that $\nabla \mathbf{g}=\mathbf{G}$ and extend it by periodicity in $\mathbb{R}^{3}$. Suppose for a moment that $a \neq-1 / 3$ and let

$$
\mathbf{g}(x):=\left\{\begin{array}{llll}
\mathbf{g}_{i}(x) & \text { on } & F_{i}, & i=1,3 \\
\mathbf{g}_{i}^{\prime}(x) & \text { on } & F_{i}^{\prime}, & i=2,4 \\
\mathbf{g}_{i}^{\prime \prime}(x) & \text { on } & F_{i}^{\prime \prime}, & i=2,4
\end{array}\right.
$$

Copyright (c) by SIAM. Unauthorized reproduction of this article is prohibited. 
where

$$
\begin{aligned}
& \mathbf{g}_{1}(x):=\left\{\begin{array}{c}
a x_{1}+2 G_{a, c} x_{3}, \\
-2 G_{a, b} x_{1}+b x_{2}-2 G_{b, c} x_{3}, \\
c x_{3},
\end{array} \quad \mathbf{g}_{3}(x):=\left\{\begin{array}{c}
a x_{1}-2 G_{a, c} x_{3}, \\
-2 G_{a, b} x_{1}+b x_{2}+2 G_{b, c} x_{3}, \\
c x_{3},
\end{array}\right.\right. \\
& \mathbf{g}_{2}^{\prime}(x):=\left\{\begin{array}{c}
a x_{1}+2 G_{a, c} x_{3}, \\
2 G_{a, b} x_{1}+b x_{2}+2 G_{b, c} x_{3}, \\
c x_{3},
\end{array} \quad \mathbf{g}_{4}^{\prime}(x):=\left\{\begin{array}{c}
a x_{1}-2 G_{a, c} x_{3}, \\
2 G_{a, b} x_{1}+b x_{2}-2 G_{b, c} x_{3}, \\
c x_{3},
\end{array}\right.\right. \\
& \mathbf{g}_{2}^{\prime \prime}(x):=\left\{\begin{array}{c}
a x_{1}-2 G_{a, c} x_{3}, \\
2 G_{a, b}\left(x_{1}-2 T\right)+b x_{2}+2 G_{b, c} x_{3}, \quad G_{a, c} x_{3}, \\
c x_{3},
\end{array} \quad:=\left\{\begin{array}{c}
\mathbf{g}_{4}^{\prime \prime}(x) \\
2 G_{a, b}\left(x_{1}-2 T\right)+b x_{2}-2 G_{b, c} x_{3}, \\
c x_{3} .
\end{array}\right.\right.
\end{aligned}
$$

Then, in the case $a=-1 / 3$, let

$$
\mathbf{g}(x):=\left\{\begin{array}{lll}
\mathbf{g}_{5}(x) & \text { on } & F_{5} \\
\mathbf{g}_{6}(x) & \text { on } & F_{6}
\end{array}\right.
$$

where

$$
\mathbf{g}_{5}(x):=\left\{\begin{array}{c}
-\frac{1}{3} x_{1}, \\
b x_{2}-2 G_{b, c} x_{3}, \\
c x_{3},
\end{array} \quad \mathbf{g}_{6}(x):=\left\{\begin{array}{c}
-\frac{1}{3} x_{1}, \\
b x_{2}+2 G_{b, c} x_{3} \\
c x_{3} .
\end{array}\right.\right.
$$

Now, let us define $\widetilde{\mathbf{g}}$ as the extension of $\mathbf{g}(x)$ in $\mathbb{R}^{3}$ by periodicity, $\mathbf{f}(x):=\mathbf{R} \widetilde{\mathbf{g}}\left(\mathbf{R}^{T} x\right)$ and

$$
\mathbf{f}_{n}(x):=\frac{1}{n} \mathbf{f}\left(n x_{1}, n x_{2}, n x_{3}\right), \quad n \in \mathbb{N} .
$$

It is straightforward to prove that $\widetilde{\mathbf{g}}, \mathbf{f}$, and $\mathbf{f}_{n}$ are Lipschitz-continuous functions (in this regard, observe that $\mathbf{G}(x)$ satisfies the Legendre-Hadamard condition [27]). Moreover, we note that $\nabla \mathbf{f}_{n}=\mathbf{F}_{n}$ and that $\left\{\mathbf{f}_{n}\right\}$ converges to $(\mathbf{Q} x)$ uniformly on compact sets as $n \rightarrow \infty$. Finally, in order to fulfill the condition

$$
\operatorname{support}\left(\mathbf{u}_{n}-\mathbf{u}\right) \subset \subset U \text {, }
$$

we modify slightly the construction above. For any positive $\varepsilon$ define the compact set $^{1}$

$$
K:=\left\{x \in U: \operatorname{dist}\left(x, U^{c}\right) \leq \varepsilon\right\} .
$$

Let $\theta(x) \in C_{c}^{\infty}(U)$ be a scalar test function with support $K^{\prime}:=\left\{x \in U: \operatorname{dist}\left(x, U^{c}\right) \leq\right.$ $\varepsilon / 2\}$, such that $\theta(x) \equiv 1$ on $K$ and $0 \leq \theta(x) \leq 1$ (see also Figure 2 (right)) and let

$$
\mathbf{u}_{n}(x):=\mathbf{u}(x)+\gamma \theta(x)\left(\mathbf{f}_{n}(x)-\mathbf{Q} x\right) .
$$

By construction, it follows that $\mathbf{u}_{n} \rightarrow \mathbf{u}$ in $H^{1}\left(U, \mathbb{R}^{3}\right)$ and $\operatorname{supp}\left(\mathbf{u}_{n}-\mathbf{u}\right) \subset \subset U$.

\footnotetext{
${ }^{1} K$ is nonempty provided, of course, that $\varepsilon$ is not too large. In what follows we implicitly assume $\varepsilon$ such that $K$ is nonempty.
}

Copyright $@$ by SIAM. Unauthorized reproduction of this article is prohibited. 
We are in a position to prove that the sequence $\left\{\mathbf{Q}_{n}, \mathbf{u}_{n}\right\} \subset L^{2}\left(\Omega, \mathcal{Q}_{F r}\right) \times H^{1}\left(\Omega, \mathbb{R}^{3}\right)$ defined in (2.14) and (2.18) yields (2.7). Observe that, since $\mathbf{Q}_{n}$ ranges in $\mathcal{Q}_{F r}$ for any $n \in \mathbb{N}$, then $\mathrm{F}_{F r, m e c}\left(\mathbf{Q}_{n}, \mathbf{u}_{n}\right)<+\infty$ and we can write

$$
\int_{U} f_{m e c}\left(\mathbf{Q}_{n}, \nabla \mathbf{u}_{n}\right) d x=\int_{K} f_{m e c}\left(\mathbf{Q}_{n}, \nabla \mathbf{u}_{n}\right) d x+\int_{U \backslash K} f_{m e c}\left(\mathbf{Q}_{n}, \nabla \mathbf{u}_{n}\right) d x .
$$

Consider the first summand on the right-hand side of (2.19). Recalling that $\operatorname{tr} \mathbf{F}_{n}=$ $\operatorname{tr} \mathbf{Q}_{n}=\operatorname{tr} \mathbf{Q}=0$, we have

$$
\begin{aligned}
& \int_{K} f_{m e c}\left(\mathbf{Q}_{n}, \nabla \mathbf{u}+\gamma \mathbf{F}_{n}-\gamma \mathbf{Q}\right) d x=\int_{K}\left\{\mu\left|\gamma \mathbf{Q}_{n}-\mathbb{E}(\nabla \mathbf{u})-\gamma \mathbf{Q}_{n}+\gamma \mathbf{Q}\right|^{2}\right. \\
+ & \left.\frac{\lambda}{2}\left(\operatorname{tr}\left(\nabla \mathbf{u}+\gamma \mathbf{F}_{n}-\gamma \mathbf{Q}-\gamma \mathbf{Q}_{n}\right)\right)^{2}\right\} d x=\int_{K}\left(\mu|\mathbb{E}(\nabla \mathbf{u})-\gamma \mathbf{Q}|^{2}+\frac{\lambda}{2}(\operatorname{div} \mathbf{u})^{2}\right) d x .
\end{aligned}
$$

We turn to the second summand on the right-hand side of (2.19). In view of the growth condition in (1.21), we can write (in what follows the constants may change from line to line while we maintain the same name)

$$
\begin{array}{r}
\int_{U \backslash K} f_{m e c}\left(\mathbf{Q}_{n}, \nabla \mathbf{u}+\gamma \theta\left(\mathbf{F}_{n}-\mathbf{Q}\right)+\gamma\left(\nabla \theta \otimes\left(\mathbf{f}_{n}-\mathbf{Q} x\right)^{T}\right)\right) d x \\
\leq \int_{U \backslash K} \operatorname{Const}\left\{1+|\mathbb{E}(\nabla \mathbf{u})|^{2}+\gamma^{2}\left|\mathbf{Q}_{n}-\mathbf{Q}\right|^{2}+\gamma^{2}|\nabla \theta|^{2}\left|\mathbf{f}_{n}-\mathbf{Q} x\right|^{2}\right\} d x .
\end{array}
$$

Recalling that $\nabla \theta$ is bounded and that $\mathbf{f}_{n}(x)$ converges uniformly to $(\mathbf{Q} x)$, if we take the limit in (2.19), we have

$$
\begin{aligned}
\limsup _{n \rightarrow+\infty} \int_{U} f_{m e c}\left(\mathbf{Q}_{n}, \nabla \mathbf{u}_{n}\right) d x & \leq \limsup _{n \rightarrow+\infty} \int_{K} f_{m e c}\left(\mathbf{Q}_{n}, \nabla \mathbf{u}_{n}\right) d x+\text { Const } \frac{\varepsilon}{m} \\
& =\int_{K} f_{m e c}(\mathbf{Q}, \nabla \mathbf{u}) d x+\text { Const } \frac{\varepsilon}{m}
\end{aligned}
$$

where $m \in \mathbb{N}$ (the role of the natural number $m$ will be clear in the next paragraph). Since $f_{m e c}$ is nonnegative we can enlarge $K$ to $U$,

$$
\limsup _{n \rightarrow+\infty} \int_{U} f_{m e c}\left(\mathbf{Q}_{n}, \nabla \mathbf{u}_{n}\right) d x \leq \int_{U} f_{m e c}(\mathbf{Q}, \nabla \mathbf{u}) d x+\text { Const } \frac{\varepsilon}{m} .
$$

Noticing that the constant is independent of $\varepsilon$, in what follows we simply write $\frac{\varepsilon}{m}$ instead of Const $\frac{\varepsilon}{m}$. In the next paragraph we show that the result above suffices to prove $\overline{\mathrm{F}}_{F r, m e c} \leq \mathrm{F}_{B, \text { mec }}$ (up to an error of order $\varepsilon$ ) under the additional assumption that $\mathbf{Q}: \Omega \rightarrow \mathcal{Q}_{B}$ is piecewise-constant.

Step 2, $\mathbf{Q}(x)$ piecewise-constant. Suppose $\mathbf{Q} \in L^{2}\left(\Omega, \mathcal{Q}_{B}\right)$ and piecewiseconstant, that is, there exists a partition of $\Omega$ consisting of a finite number $m$ of open and pairwise disjoint sets $\Omega^{j}$ such that

$$
\Omega=\bigcup_{j=1}^{m} \Omega^{j} \cup N,
$$

where $|N|=0$ and $\mathbf{Q}^{j}:=\left.\mathbf{Q}\right|_{\Omega^{j}}$ is constant and biaxial. By Step 1 it follows that, for every $\Omega^{j}$, there exist sequences

$$
\mathbf{Q}_{n}^{j} \rightarrow \mathbf{Q}^{j} \text { in } L^{2}\left(\Omega^{j}, \mathbb{M}^{3 \times 3}\right), \quad \mathbf{u}_{n}^{j} \rightarrow \mathbf{u}^{j} \text { in } H^{1}\left(\Omega^{j}, \mathbb{R}^{3}\right) \quad \text { as } n \rightarrow+\infty,
$$


with $\operatorname{supp}\left(\mathbf{u}_{n}^{j}-\mathbf{u}_{j}\right) \subset \subset \Omega^{j}$ and where $\mathbf{u}^{j}$ is the restriction of $\mathbf{u}$ on $\Omega^{j}$, such that

$$
\limsup _{n \rightarrow+\infty} \int_{\Omega^{j}} f_{m e c}\left(\mathbf{Q}_{n}^{j}, \nabla \mathbf{u}_{n}^{j}\right) d x \leq \int_{\Omega^{j}} f_{m e c}\left(\mathbf{Q}^{j}, \nabla \mathbf{u}^{j}\right) d x+\frac{\varepsilon}{m} .
$$

Define

$$
\widetilde{\mathbf{u}}_{n}:=\mathbf{u}_{n}^{j} \text { on } \Omega^{j}, \quad \widetilde{\mathbf{Q}}_{n}:=\mathbf{Q}_{n}^{j} \text { on } \Omega^{j}, \quad j=1, \ldots, m .
$$

Recalling that support $\left(\mathbf{u}_{n}^{j}-\mathbf{u}^{j}\right) \subset \subset \Omega^{j}$, then $\widetilde{\mathbf{u}}_{n} \in H^{1}\left(\Omega, \mathbb{R}^{3}\right)$ and $\widetilde{\mathbf{u}}_{n}-\mathbf{u} \in H_{o}^{1}\left(\Omega, \mathbb{R}^{3}\right)$. Therefore, we can write

$$
\begin{aligned}
& \liminf _{n \rightarrow+\infty} \int_{\Omega} f_{m e c}\left(\widetilde{\mathbf{Q}}_{n}, \nabla \widetilde{\mathbf{u}}_{n}\right) d x=\liminf _{n \rightarrow+\infty} \sum_{j=1}^{m} \int_{\Omega^{j}} f_{m e c}\left(\mathbf{Q}_{n}^{j}, \nabla \mathbf{u}_{n}^{j}\right) d x \\
\leq & \sum_{j=1}^{m} \limsup _{n \rightarrow+\infty} \int_{\Omega^{j}} f_{m e c}\left(\mathbf{Q}_{n}^{j}, \nabla \mathbf{u}_{n}^{j}\right) d x \leq \sum_{j=1}^{m}\left(\int_{\Omega^{j}} f_{m e c}\left(\mathbf{Q}^{j}, \nabla \mathbf{u}^{j}\right) d x+\frac{\varepsilon}{m}\right) \\
= & \sum_{j=1}^{m} \int_{\Omega^{j}} f_{m e c}\left(\mathbf{Q}^{j}, \nabla \mathbf{u}^{j}\right) d x+\varepsilon=\int_{\Omega} f_{m e c}(\mathbf{Q}, \nabla \mathbf{u}) d x+\varepsilon,
\end{aligned}
$$

where the inequality in (2.27) follows from (2.25). Summarizing, we have

$$
\begin{aligned}
\overline{\mathrm{F}}_{F r, m e c}(\mathbf{Q}, \mathbf{u}) & \leq \liminf _{n \rightarrow+\infty} \int_{\Omega} f_{m e c}\left(\widetilde{\mathbf{Q}}_{n}, \nabla \widetilde{\mathbf{u}}_{n}\right) d x \\
& \leq \int_{\Omega} f_{m e c}(\mathbf{Q}, \nabla \mathbf{u}) d x+\varepsilon=\mathrm{F}_{B, m e c}(\mathbf{Q}, \mathbf{u})+\varepsilon
\end{aligned}
$$

We can now extend (2.28) to the case in which $\mathbf{Q}$ is any tensor in $L^{2}\left(\Omega, \mathcal{Q}_{B}\right)$.

Step 3, $\mathbf{Q}(\boldsymbol{x}) \in \boldsymbol{L}^{\mathbf{2}}\left(\boldsymbol{\Omega}, \mathcal{Q}_{B}\right)$. Let any $(\mathbf{Q}, \mathbf{u}) \in L^{2}\left(\Omega, \mathcal{Q}_{B}\right) \times H^{1}\left(\Omega, \mathbb{R}^{3}\right)$. By applying Proposition 3 with $d=3$ and $\mathcal{K}=\mathcal{Q}_{B}$ there exists a sequence $\left\{\mathbf{Q}_{k}\right\} \subset$ $L^{2}\left(\Omega, \mathcal{Q}_{B}\right)$ of piecewise-constant matrices such that

$$
\mathbf{Q}_{k} \rightarrow \mathbf{Q} \text { in } L^{2}\left(\Omega, \mathbb{M}^{3 \times 3}\right) \text { as } k \rightarrow+\infty .
$$

Therefore, if we plug $\mathbf{Q}_{k}$ into (2.28), we obtain

$$
\overline{\mathrm{F}}_{F r, m e c}\left(\mathbf{Q}_{k}, \mathbf{u}\right) \leq \int_{\Omega} f_{m e c}\left(\mathbf{Q}_{k}, \nabla \mathbf{u}\right) d x+\varepsilon,
$$

and, taking the limit in $k$,

$$
\overline{\mathrm{F}}_{F r, m e c}(\mathbf{Q}, \mathbf{u}) \leq \liminf _{k \rightarrow+\infty} \overline{\mathrm{F}}_{F r, m e c}\left(\mathbf{Q}_{k}, \mathbf{u}\right) \leq \liminf _{k \rightarrow+\infty} \int_{\Omega} f_{m e c}\left(\mathbf{Q}_{k}, \nabla \mathbf{u}\right) d x+\varepsilon .
$$

By the continuity of the integral on the right-hand side in (2.31) we have

$$
\overline{\mathrm{F}}_{F r, m e c}(\mathbf{Q}, \mathbf{u}) \leq \int_{\Omega} f_{m e c}(\mathbf{Q}, \nabla \mathbf{u}) d x+\varepsilon
$$

and, taking the limit $\varepsilon \rightarrow 0$,

$$
\overline{\mathrm{F}}_{F r, m e c}(\mathbf{Q}, \mathbf{u}) \leq \mathrm{F}_{B, m e c}(\mathbf{Q}, \mathbf{u}) .
$$


Remark 2. In view of the proof of Theorem 1 it is an easy exercise to show that $L^{2}\left(\Omega, \mathcal{Q}_{B}\right)$ coincides with $\overline{c o}\left(L^{2}\left(\Omega, \mathcal{Q}_{F r}\right)\right)$, the closed convex hull of $L^{2}\left(\Omega, \mathcal{Q}_{F r}\right)$ with respect to the weak topology. For the reader's convenience, we sketch the argument required for the proof of this claim. The argument of Step 3 ensures that any $\mathbf{Q} \in$ $L^{2}\left(\Omega, \mathcal{Q}_{B}\right)$ can be approximated (in the strong topology) by a sequence of piecewiseconstant tensors in $L^{2}\left(\Omega, \mathcal{Q}_{B}\right)$. Moreover, the constructions contained in Steps 1 and 2 prove that it is possible to approximate any piecewise-constant matrix in $L^{2}\left(\Omega, \mathcal{Q}_{B}\right)$ with a weakly convergent sequence of Frank-type tensors. This is sufficient to prove that

$$
L^{2}\left(\Omega, \mathcal{Q}_{B}\right) \subseteq \overline{c o}\left(L^{2}\left(\Omega, \mathcal{Q}_{F r}\right)\right)
$$

The opposite inclusion is trivial because $L^{2}\left(\Omega, \mathcal{Q}_{B}\right)$ is closed, convex, and contains $L^{2}\left(\Omega, \mathcal{Q}_{F r}\right)$ by definition. Moreover, since $L^{2}\left(\Omega, \mathcal{Q}_{F r}\right) \subset L^{2}\left(\Omega, \mathcal{Q}_{U}\right) \subset L^{2}\left(\Omega, \mathcal{Q}_{B}\right)$, it also follows that $\overline{c o}\left(L^{2}\left(\Omega, \mathcal{Q}_{U}\right)\right)=L^{2}\left(\Omega, \mathcal{Q}_{B}\right)$.

Remark 3 (boundary conditions). Theorem 1 can be improved to obtain relaxation results in the presence of slightly different boundary conditions for the displacement $\mathbf{u}$, as shown in the next corollary.

Corollary 1. Let $\Omega \subset \mathbb{R}^{3}$ be an open, bounded, connected set with Lipschitz boundary, and let $\Gamma_{D} \subseteq \partial \Omega$ be an open subset with positive surface measure. Take $\mathbf{g}(x) \in H^{1}\left(\Omega, \mathbb{R}^{3}\right)$ and assume $f_{\text {mec }}$ as in (1.20). Define

$$
F_{X, m e c}^{\Gamma_{D}, g}(\mathbf{Q}, \mathbf{u}):=\left\{\begin{array}{cc}
\int_{\Omega} f_{m e c}(\mathbf{Q}, \nabla \mathbf{u}) d x & \text { on } L^{2}\left(\Omega, \mathcal{Q}_{X}\right) \times H_{\Gamma_{D}}^{1}\left(\Omega, \mathbb{R}^{3}\right)+\mathbf{g}(x), \\
+\infty & \text { otherwise in } L^{2}\left(\Omega, \mathbb{M}^{3 \times 3}\right) \times H^{1}\left(\Omega, \mathbb{R}^{3}\right),
\end{array}\right.
$$

where $X$ stands for either $F r$, or $U$, or $B$. Then the relaxation of $\mathrm{F}_{X, m e c}^{\Gamma_{D}, g}$ in the sense of $\sigma$ (see (2.1)) is $\mathrm{F}_{B, m e c}^{\Gamma_{D}, g}$.

Proof. Consider first $\Gamma_{D}=\partial \Omega$. We can repeat the proof of Theorem 1 even in this case, because the sequence $\left\{\widetilde{\mathbf{u}}_{n}\right\}$ defined in Step 2 is such that $\widetilde{\mathbf{u}}_{n}-\mathbf{u} \in H_{o}^{1}\left(\Omega, \mathbb{R}^{3}\right)$ for every $n \in \mathbb{N}$. Then the proof for any $\Gamma_{D} \subset \partial \Omega$ follows from a standard argument. Observe that we can write

$$
\mathrm{F}_{F r, m e c}(\mathbf{Q}, \mathbf{u}) \leq \mathrm{F}_{F r, m e c}^{\Gamma_{D}, g}(\mathbf{Q}, \mathbf{u}) \leq \mathrm{F}_{F r, m e c}^{\partial \Omega, \widehat{g}}(\mathbf{Q}, \mathbf{u}),
$$

where $\widehat{\mathbf{g}} \equiv \mathbf{u}$. By relaxing all the functionals in (2.34), we show that the relaxation of $\mathrm{F}_{F r, m e c}^{\Gamma_{D}, g}$ is equal to $\mathrm{F}_{B, \text { mec }}$ if $(\mathbf{Q}, \mathbf{u})$ belongs to the set

$$
L^{2}\left(\Omega, \mathcal{Q}_{B}\right) \times H_{\Gamma_{D}}^{1}\left(\Omega, \mathbb{R}^{3}\right)+\mathbf{g}(x), \operatorname{div} \mathbf{u}=0
$$

and $+\infty$ in the complementary of $(2.35)$ in $L^{2}\left(\Omega, \mathbb{M}^{3 \times 3}\right) \times H^{1}\left(\Omega, \mathbb{R}^{3}\right)$, because (2.35) is closed in the sense of $\sigma$.

2.1.2. The case of incompressible elastomers. We turn to the relaxation of models for incompressible elastomers, that is, the case in which we assign an infinite penalization if the displacement $\mathbf{u}$ is not divergence-free. In the next theorem we recall the definition of the functional $\mathcal{F}_{X, \text { mec }}$ which was introduced at the beginning of section 2 .

Theorem 2. Let $\Omega \subset \mathbb{R}^{3}$ be an open, bounded, connected set with Lipschitz boundary. Assume $f_{m e c}$ as in (1.20) and define

$$
\mathcal{F}_{X, m e c}(\mathbf{Q}, \mathbf{u}):=\left\{\begin{array}{cc}
\int_{\Omega} f_{m e c}(\mathbf{Q}, \nabla \mathbf{u}) d x & \text { on } L^{2}\left(\Omega, \mathcal{Q}_{X}\right) \times H^{1}\left(\Omega, \mathbb{R}^{3}\right), \operatorname{div} \mathbf{u}=0 \\
+\infty & \text { otherwise in } L^{2}\left(\Omega, \mathbb{M}^{3 \times 3}\right) \times H^{1}\left(\Omega, \mathbb{R}^{3}\right)
\end{array}\right.
$$

Copyright (c) by SIAM. Unauthorized reproduction of this article is prohibited. 
where $X$ stands for either $F r$, or $U$, or $B$. Then the relaxation of $\mathcal{F}_{X, \text { mec }}$ in the sense of $\sigma$ (see (2.1)) is $\mathcal{F}_{B, m e c}$.

Proof. Again, in the case $X=B$ there is nothing to prove and the discussion of the case $X=U$ is postponed to section 2.2.1. In the case $X=F r$ the proof is essentially based on an argument of Braides [5] and largely employed also in [7]. We show that $\overline{\mathcal{F}}_{F r, \text { mec }}=\mathcal{F}_{B, \text { mec }}$ by proving two inequalities. First of all, by definition it follows that $\mathcal{F}_{B, \text { mec }} \leq \mathcal{F}_{F r, \text { mec }}$ and, by relaxing both sides, we obtain $\mathcal{F}_{B, \text { mec }} \leq \overline{\mathcal{F}}_{F r, m e c}$. We are left with the proof of the opposite inequality

$$
\overline{\mathcal{F}}_{F r, m e c} \leq \mathcal{F}_{B, m e c} .
$$

For convenience, we label with $\mathrm{F}_{X, \text { mec }}^{h}$ the functional introduced in (2.4), that is,

$$
\mathrm{F}_{X, m e c}^{h}(\mathbf{Q}, \mathbf{u})=\left\{\begin{array}{l}
\int_{\Omega}\left(\mu|\mathbb{E}(\nabla \mathbf{u})-\gamma \mathbf{Q}|^{2}+\frac{h}{2}(\operatorname{div} \mathbf{u})^{2}\right) d x \text { on } L^{2}\left(\Omega, \mathcal{Q}_{X}\right) \times H^{1}\left(\Omega, \mathbb{R}^{3}\right), \\
+\infty \\
\text { otherwise in } L^{2}\left(\Omega, \mathbb{M}^{3 \times 3}\right) \times H^{1}\left(\Omega, \mathbb{R}^{3}\right),
\end{array}\right.
$$

where $X$ stands for either $F r$ or $B$, and $h \in \mathbb{N}$. Hence, by Theorem 1, the relaxation of $\mathrm{F}_{X, m e c}^{h}$ is $\mathrm{F}_{B, m e c}^{h}$. The following chain of equalities has a crucial role in order to prove (2.36):

$$
\begin{aligned}
\Gamma-\lim _{h \rightarrow+\infty} \mathrm{F}_{F r, m e c}^{h}(\mathbf{Q}, \mathbf{u}) & =\sup _{h} \overline{\mathrm{F}}_{F r, m e c}^{h}(\mathbf{Q}, \mathbf{u}) \\
& =\sup _{h} \mathrm{~F}_{B, m e c}^{h}(\mathbf{Q}, \mathbf{u})=\mathcal{F}_{B, m e c}(\mathbf{Q}, \mathbf{u}) .
\end{aligned}
$$

Notice that the first equality in (2.37) follows from Proposition 1 and the second one is a consequence of Theorem 1. Then the last equality is an application of the Beppo-Levi theorem for monotone sequences of integrals, stating that the supremum of a family of increasing integrals coincides with the integral of the pointwise limit of the energy densities:

$$
\lim _{h \rightarrow+\infty}\left(\mu|\mathbb{E}(\mathbf{F})-\gamma \mathbf{Q}|^{2}+\frac{h}{2}(\operatorname{tr} \mathbf{F})^{2}\right)=\left\{\begin{array}{cc}
\mu|\mathbb{E}(\mathbf{F})-\gamma \mathbf{Q}|^{2} & \text { if } \operatorname{tr} \mathbf{F}=0 \\
+\infty & \text { otherwise }
\end{array}\right.
$$

Now, if $\operatorname{div} \mathbf{u} \neq 0$ or $\mathbf{Q} \notin L^{2}\left(\Omega, \mathcal{Q}_{B}\right)$ the inequality $\overline{\mathcal{F}}_{F r, \text { mec }} \leq \mathcal{F}_{B, \text { mec }}$ is trivial, and for the rest of the proof we can suppose $\operatorname{div} \mathbf{u}=0$ and $\mathbf{Q} \in L^{2}\left(\Omega, \mathcal{Q}_{B}\right)$. Since the Gamma-convergence is metrizable, it is sufficient to prove that for any sequence $\left\{\mathbf{Q}_{h}, \mathbf{u}_{h}\right\} \subset L^{2}\left(\Omega, \mathcal{Q}_{B}\right) \times H^{1}\left(\Omega, \mathbb{R}^{3}\right)$ such that

$$
\mathbf{Q}_{h}, \mathbf{u}_{h} \stackrel{\sigma}{\rightarrow} \mathbf{Q}, \mathbf{u} \quad \text { as } h \rightarrow+\infty,
$$

there exists a sequence $\mathbf{z}_{h} \rightarrow \mathbf{u}$ in $H^{1}\left(\Omega, \mathbb{R}^{3}\right)$ with $\operatorname{div} \mathbf{z}_{h}=0$ such that

$$
\liminf _{h \rightarrow+\infty} \mathcal{F}_{F r, m e c}\left(\mathbf{Q}_{h}, \mathbf{z}_{h}\right) \leq \liminf _{h \rightarrow+\infty} \mathrm{F}_{F r, m e c}^{h}\left(\mathbf{Q}_{h}, \mathbf{u}_{h}\right) .
$$

In view of (2.37), by taking the infimum over all the sequences $\left\{\mathbf{Q}_{h}, \mathbf{u}_{h}\right\}$ which converge to $(\mathbf{Q}, \mathbf{u})$ with respect to the topology $\sigma$, we obtain $\mathcal{F}_{B, \text { mec }}$ on the right-hand side of (2.39). Moreover, by applying Corollary 1 with $\Gamma_{D}=\partial \Omega$, we may restrict ourselves to sequences of displacements such that $\mathbf{u}_{h}-\mathbf{u} \in H_{o}^{1}\left(\Omega, \mathbb{R}^{3}\right)$. In order to 
define $\left\{\mathbf{z}_{h}\right\}$, we apply Proposition 2 with $p=2, n=3$. For any $h \in \mathbb{N}$, let $\mathbf{w}_{h}(x)$ be a solution to the problem

$$
\left\{\begin{array}{c}
\mathbf{w}_{h} \in H_{o}^{1}\left(\Omega, \mathbb{R}^{3}\right) \\
\operatorname{div} \mathbf{w}_{h}=\operatorname{div} \mathbf{u}_{h}=\operatorname{div}\left(\mathbf{u}_{h}-\mathbf{u}\right), \\
\left\|\mathbf{w}_{h}\right\|_{H^{1}\left(\Omega, \mathbb{R}^{3}\right)} \leq C(\Omega)\left\|\operatorname{div} \mathbf{u}_{h}\right\|_{L^{2}(\Omega)}
\end{array}\right.
$$

Since $\mathcal{F}_{B, \text { mec }}(\mathbf{Q}, \mathbf{u})<+\infty$, we can suppose that $\mathrm{F}_{F r, m e c}^{h}\left(\mathbf{Q}_{h}, \mathbf{u}_{h}\right) \leq$ Const for every $h \in \mathbb{N}$. Hence,

$$
\left\|\operatorname{div} \mathbf{u}_{h}\right\|_{L^{2}}^{2} \leq \text { Const } / h
$$

and, by Proposition 2,

$$
\mathbf{w}_{h} \rightarrow 0 \text { in } H^{1}\left(\Omega, \mathbb{R}^{3}\right) \text { as } h \rightarrow \infty .
$$

If we let $\mathbf{z}_{h}:=\mathbf{u}_{h}-\mathbf{w}_{h}$, we have

$$
\mathbf{z}_{h} \rightarrow \mathbf{u} \text { in } H^{1}\left(\Omega, \mathbb{R}^{3}\right), \mathbf{z}_{h}-\mathbf{u}_{h} \in H_{o}^{1}\left(\Omega, \mathbb{R}^{3}\right), \text { and } \operatorname{div} \mathbf{z}_{h}=0 .
$$

Now, by Hölder's inequality it follows that

$$
\left|\int_{\Omega}\left(\left|\mathbb{E}\left(\nabla \mathbf{u}_{h}\right)-\gamma \mathbf{Q}_{h}\right|^{2}-\left|\mathbb{E}\left(\nabla \mathbf{z}_{h}\right)-\gamma \mathbf{Q}_{h}\right|^{2}\right) d x\right| \leq \operatorname{Const}\left\|\mathbb{E}\left(\nabla \mathbf{w}_{h}\right)\right\|_{L^{2}\left(\Omega, \mathbb{M}^{3 \times 3}\right)},
$$

and, in conclusion,

$$
\begin{aligned}
\liminf _{h \rightarrow+\infty} \mathcal{F}_{F r, m e c}\left(\mathbf{Q}_{h}, \mathbf{z}_{h}\right)= & \liminf _{h \rightarrow+\infty} \int_{\Omega} \mu\left|\mathbb{E}\left(\nabla \mathbf{z}_{h}\right)-\gamma \mathbf{Q}_{h}\right|^{2} d x \\
\leq & \liminf _{h \rightarrow+\infty} \int_{\Omega} \mu\left|\mathbb{E}\left(\nabla \mathbf{u}_{h}\right)-\gamma \mathbf{Q}_{h}\right|^{2} d x \\
& +\lim _{h \rightarrow+\infty}\left|\int_{\Omega} \mu\left(\left|\mathbb{E}\left(\nabla \mathbf{z}_{h}\right)-\gamma \mathbf{Q}_{h}\right|^{2}-\left|\mathbb{E}\left(\nabla \mathbf{u}_{h}\right)-\gamma \mathbf{Q}_{h}\right|^{2}\right) d x\right| \\
= & \liminf _{h \rightarrow+\infty} \int_{\Omega} \mu\left|\mathbb{E}\left(\nabla \mathbf{u}_{h}\right)-\gamma \mathbf{Q}_{h}\right|^{2} d x \\
& +0 \leq \liminf _{h \rightarrow+\infty} \mathrm{F}_{F r, \text { mec }}^{h}\left(\mathbf{Q}_{h}, \mathbf{u}_{h}\right) .
\end{aligned}
$$

Remark 4. The relaxation result for incompressible rubbers holds also in the presence of slightly different boundary conditions for the displacement $\mathbf{u}$, as shown in the next corollary.

Corollary 2. Let $\Omega \subset \mathbb{R}^{3}$ be an open, bounded, connected set with Lipschitz boundary, and let $\Gamma_{D} \subseteq \partial \Omega$ be an open subset with positive surface measure. Take $\mathbf{g}(x) \in H^{1}\left(\Omega, \mathbb{R}^{3}\right)$ with $\operatorname{div} \mathbf{g}(x)=0$ a.e. in $\Omega, f_{\text {mec }}$ as in (1.20) and define

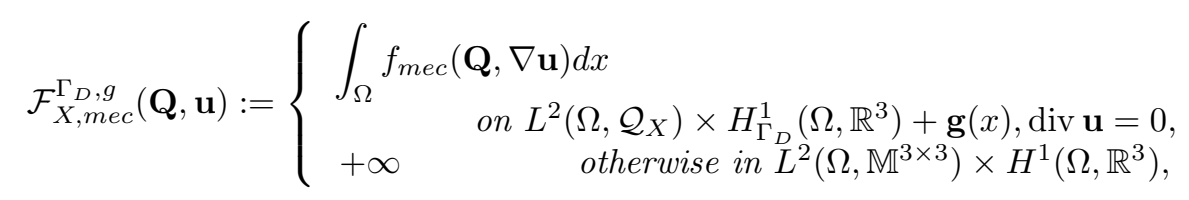

where $X$ stands for either $F r$, or $U$, or $B$. Then the relaxation of $\mathcal{F}_{X, m e c}^{\Gamma_{D}, g}$ in the sense of $\sigma$ (see (2.1)) is $\mathcal{F}_{B, \text { mec }}^{\Gamma_{D}, g}$.

Copyright $@$ by SIAM. Unauthorized reproduction of this article is prohibited. 
Proof. To start, let $\Gamma_{D}=\partial \Omega$ and $\mathbf{g}(x) \equiv 0$. The result is immediate if we assume $\mathbf{u} \in H_{o}^{1}\left(\Omega, \mathbb{R}^{3}\right)$ in the proof of Theorem 2. Then let $\mathbf{g} \in H^{1}\left(\Omega, \mathbb{R}^{3}\right)$ with $\operatorname{div} \mathbf{g}=0$. In this case we can repeat the proof of Theorem 2 by simply considering a sequence $\left\{\mathbf{u}_{h}\right\}$ such that $\mathbf{u}_{h}-\mathbf{g} \in H_{o}^{1}\left(\Omega, \mathbb{R}^{3}\right)$ for any $h \in \mathbb{N}$. Then in the general case with any $\Gamma_{D} \subset \partial \Omega$ we can proceed exactly as in the proof of Corollary 1 where an analogous case is treated.

Remark 5. Another by-product of Theorem 2 is implicitly given by (2.37). Notice that this formula holds trivially for the energies defined for $X=B$ since $\mathrm{F}_{B, \text { mec }}^{h}=$ $\overline{\mathrm{F}}_{B, \text { mec }}^{h}$. This proves that the functional $\mathcal{F}_{B, \text { mec }}$ of an incompressible material can be approximated in the sense of Gamma-convergence by a sequence of energies with increasing bulk moduli

$$
\Gamma-\lim _{h \rightarrow+\infty} \mathrm{F}_{B, m e c}^{h}(\mathbf{Q}, \mathbf{u})=\mathcal{F}_{B, m e c}(\mathbf{Q}, \mathbf{u}) .
$$

2.2. Gamma-convergence theorem. For the reader's convenience, in the next theorem we recall the definition of $\mathcal{F}_{X, \varepsilon}(\mathbf{Q}, \mathbf{u})$.

TheOREM 3. Let $\Omega \subset \mathbb{R}^{3}$ be an open, bounded, connected set with Lipschitz boundary, and let $\Gamma_{D} \subseteq \partial \Omega$ be an open subset with positive surface measure. Take $\mathbf{g}(x) \in H^{1}\left(\Omega, \mathbb{R}^{3}\right)$ with $\operatorname{div} \mathbf{g}(x)=0$ a.e. in $\Omega$ and assume $f_{\text {mec }}$ as in (1.20). Define

$$
\mathcal{F}_{X, \varepsilon}(\mathbf{Q}, \mathbf{u}):=\left\{\begin{array}{rr}
\int_{\Omega}\left(\varepsilon^{2}|\nabla \mathbf{Q}|^{2}+f_{m e c}(\mathbf{Q}, \nabla \mathbf{u})\right) d x \\
+\infty \quad \begin{array}{l}
\text { on } H^{1}\left(\Omega, \mathcal{Q}_{X}\right) \times H^{1}\left(\Omega, \mathbb{R}^{3}\right), \operatorname{div} \mathbf{u}=0, \\
\text { otherwise in } L^{2}\left(\Omega, \mathbb{M}^{3 \times 3}\right) \times H^{1}\left(\Omega, \mathbb{R}^{3}\right)
\end{array}
\end{array}\right.
$$

and

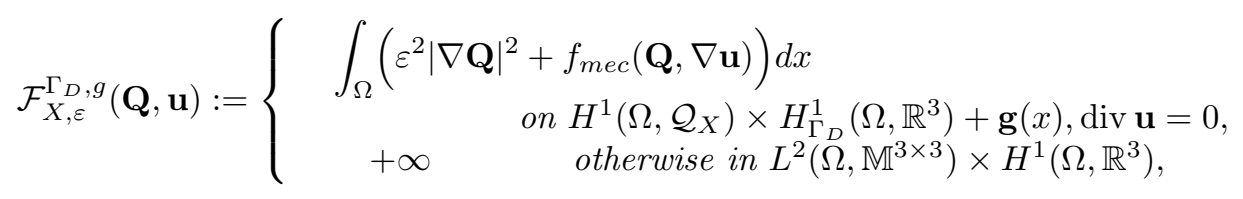

where $X$ stands for either $F r$, or $U$, or B. Then

$$
\Gamma(\sigma)-\lim _{\varepsilon \rightarrow 0} \mathcal{F}_{X, \varepsilon}(\mathbf{Q}, \mathbf{u})=\mathcal{F}_{B, m e c}(\mathbf{Q}, \mathbf{u})
$$

and

$$
\Gamma(\sigma)-\lim _{\varepsilon \rightarrow 0} \mathcal{F}_{X, \varepsilon}^{\Gamma_{D}, g}(\mathbf{Q}, \mathbf{u})=\mathcal{F}_{B, m e c}^{\Gamma_{D}, g}(\mathbf{Q}, \mathbf{u}),
$$

where $\mathcal{F}_{B, \text { mec }}$ and $\mathcal{F}_{B, \text { mec }}^{\Gamma_{D}, g}$ are defined in Theorem 2 and Corollary 2.

Proof. We prove (2.45) in the case $X=F r$. We find it convenient to characterize the Gamma-limit of a sequence of functionals by the liminf and limsup inequalities (see [4], [14]). First of all, observe that if $\mathbf{Q} \notin L^{2}\left(\Omega, \mathcal{Q}_{B}\right)$, then there is nothing to prove. Then, as $\varepsilon \rightarrow 0$, let $\left\{\varepsilon_{j}\right\}$ be a countable sequence such that $\varepsilon_{j} \rightarrow 0$ as $j \rightarrow+\infty$.

Liminf inequality. It is enough to prove that for any sequence

$$
\left\{\mathbf{Q}_{j}, \mathbf{u}_{j}\right\} \subset L^{2}\left(\Omega, \mathcal{Q}_{B}\right) \times H^{1}\left(\Omega, \mathbb{R}^{3}\right) \text { such that } \mathbf{Q}_{j}, \mathbf{u}_{j} \stackrel{\sigma}{\rightarrow} \mathbf{Q}, \mathbf{u} \text { as } j \rightarrow+\infty,
$$

we have

$$
\mathcal{F}_{B, m e c}^{\Gamma_{D}, g}(\mathbf{Q}, \mathbf{u}) \leq \liminf _{j \rightarrow+\infty} \mathcal{F}_{F r, \varepsilon_{j}}^{\Gamma_{D}, g}\left(\mathbf{Q}_{j}, \mathbf{u}_{j}\right)
$$

Copyright $@$ by SIAM. Unauthorized reproduction of this article is prohibited. 
We can restrict our attention to sequences for which the right-hand side of (2.47) is finite and, passing to subsequences (not relabeled), uniformly bounded by some positive constant $C$. Therefore, we can write

$\liminf _{j \rightarrow+\infty} \int_{\Omega} \mu\left|\mathbb{E}\left(\nabla \mathbf{u}_{j}\right)-\gamma \mathbf{Q}_{j}\right|^{2} d x \leq \liminf _{j \rightarrow+\infty} \int_{\Omega}\left(\varepsilon_{j}^{2}\left|\nabla \mathbf{Q}_{j}\right|^{2}+\mu\left|\mathbb{E}\left(\nabla \mathbf{u}_{j}\right)-\gamma \mathbf{Q}_{j}\right|^{2}\right) d x \leq C$.

By weak convergence, Korn's theorem, and the properties of the trace, the limit functional is finite over the set $L^{2}\left(\Omega, \mathcal{Q}_{B}\right) \times\left\{H_{\Gamma_{D}}^{1}\left(\Omega, \mathbb{R}^{3}\right)+\mathbf{g}(x)\right.$, div $\left.\mathbf{u}=0\right\}$ and hence (2.47) follows by Corollary 2 .

Limsup inequality. According to the sequential characterization, we introduce the Gamma-limsup as follows:

$$
\Gamma-\limsup _{j \rightarrow+\infty} \mathcal{F}_{F r, \varepsilon_{j}}^{\Gamma_{D}, g}(\mathbf{Q}, \mathbf{u})=\inf \left\{\limsup _{j \rightarrow+\infty} \mathcal{F}_{F r, \varepsilon_{j}}^{\Gamma_{D}, g}\left(\mathbf{Q}_{j}, \mathbf{u}_{j}\right), \mathbf{Q}_{j}, \mathbf{u}_{j} \stackrel{\sigma}{\rightarrow} \mathbf{Q}, \mathbf{u}\right\},
$$

with $\left\{\mathbf{Q}_{j}, \mathbf{u}_{j}\right\} \subset L^{2}\left(\Omega, \mathcal{Q}_{B}\right) \times H^{1}\left(\Omega, \mathbb{R}^{3}\right)$, and we prove that

$$
\mathcal{F}_{B, \text { mec }}^{\Gamma_{D}, g}(\mathbf{Q}, \mathbf{u}) \geq \Gamma-\limsup _{j \rightarrow+\infty} \mathcal{F}_{F r, \varepsilon_{j}}^{\Gamma_{D}, g}(\mathbf{Q}, \mathbf{u}) .
$$

Observe that if $\mathbf{u} \notin\left\{H_{\Gamma_{D}}^{1}\left(\Omega, \mathbb{R}^{3}\right)+\mathbf{g}(x), \operatorname{div} \mathbf{u}=0\right\}$, then the claim is trivially true. Now, let us assume for a moment that $(\mathbf{Q}, \mathbf{u}) \in H^{1}\left(\Omega, \mathcal{Q}_{F r}\right) \times\left\{H_{\Gamma_{D}}^{1}\left(\Omega, \mathbb{R}^{3}\right)+\mathbf{g}(x)\right.$, $\operatorname{div} \mathbf{u}=0\}$ and take the constant sequence $\left\{\widehat{\mathbf{Q}}_{j}, \widehat{\mathbf{u}}_{j}\right\}=(\mathbf{Q}, \mathbf{u})$. We have

$$
\begin{aligned}
\Gamma-\limsup _{j \rightarrow+\infty} \mathcal{F}_{F r, \varepsilon_{j}}^{\Gamma_{D}, g}(\mathbf{Q}, \mathbf{u}) & \leq \limsup _{j \rightarrow+\infty} \int_{\Omega}\left(\varepsilon_{j}^{2}\left|\nabla \widehat{\mathbf{Q}}_{j}\right|^{2}+f_{m e c}\left(\widehat{\mathbf{Q}}_{j}, \nabla \widehat{\mathbf{u}}_{j}\right)\right) d x \\
& =\int_{\Omega} f_{m e c}(\mathbf{Q}, \nabla \mathbf{u}) d x .
\end{aligned}
$$

Then, by Lemma 1 , for every $\mathbf{Q} \in L^{2}\left(\Omega, \mathcal{Q}_{F r}\right)$ there exists a sequence $\left\{\mathbf{Q}_{k}\right\} \subset$ $H^{1}\left(\Omega, \mathcal{Q}_{F r}\right)$ such that

$$
\mathbf{Q}_{k} \rightarrow \mathbf{Q} \text { in } L^{2}\left(\Omega, \mathbb{M}^{3 \times 3}\right) \quad \text { as } k \rightarrow \infty,
$$

and, if we plug $\mathbf{Q}_{k}$ instead of $\mathbf{Q}$ into (2.49), we obtain

$$
\Gamma-\limsup _{j \rightarrow+\infty} \mathcal{F}_{F r, \varepsilon_{j}}^{\Gamma_{D}, g}\left(\mathbf{Q}_{k}, \mathbf{u}\right) \leq \int_{\Omega} \mu\left|\mathbb{E}(\nabla \mathbf{u})-\gamma \mathbf{Q}_{k}\right|^{2} d x \quad \forall k \in \mathbb{N} .
$$

Recalling that the Gamma-limsup is a lower semicontinuous functional [14] and that the integral on the right-hand side of (2.50) is continuous in the strong topology of $L^{2}\left(\Omega, \mathbb{M}^{3 \times 3}\right)$, we have

$$
\begin{aligned}
\Gamma-\limsup _{j \rightarrow+\infty} \mathcal{F}_{F r, \varepsilon_{j}}^{\Gamma_{D}, g}(\mathbf{Q}, \mathbf{u}) & \leq \liminf _{k \rightarrow+\infty}\left(\Gamma-\limsup _{j \rightarrow \infty} \mathcal{F}_{F r, \varepsilon_{j}}^{\Gamma_{D}, g}\left(\mathbf{Q}_{k}, \mathbf{u}\right)\right) \\
& \leq \lim _{k \rightarrow+\infty} \int_{\Omega} \mu\left|\mathbb{E}(\nabla \mathbf{u})-\gamma \mathbf{Q}_{k}\right|^{2} d x=\int_{\Omega} \mu|\mathbb{E}(\nabla \mathbf{u})-\gamma \mathbf{Q}|^{2} d x .
\end{aligned}
$$

Summarizing, for every $(\mathbf{Q}, \mathbf{u}) \in L^{2}\left(\Omega, \mathbb{M}^{3 \times 3}\right) \times H^{1}\left(\Omega, \mathbb{R}^{3}\right)$, it follows that

$$
\Gamma-\limsup _{j \rightarrow+\infty} \mathcal{F}_{F r, \varepsilon_{j}}^{\Gamma_{D}, g}(\mathbf{Q}, \mathbf{u}) \leq \mathcal{F}_{F r, m e c}^{\Gamma_{D}, g}(\mathbf{Q}, \mathbf{u}),
$$

Copyright $@$ by SIAM. Unauthorized reproduction of this article is prohibited. 
and to conclude it is sufficient to relax both sides by applying Corollary 2 .

The proof of (2.44) in the case $X=F r$ follows as above.

Then, considering the case $X=B$, the proof of (2.45) (and of (2.44)) is even easier. The liminf inequality becomes trivial, and the limsup inequality can be modified as follows. Observe that the proof for the Frank model is based on the approximation of $L^{2}\left(\Omega, \mathcal{Q}_{F r}\right)$-tensors with $H^{1}\left(\Omega, \mathcal{Q}_{F r}\right)$-tensors in the strong $L^{2}\left(\Omega, \mathbb{M}^{3 \times 3}\right)$-topology. In the biaxial case, the result can be obtained by approximating $L^{2}\left(\Omega, \mathcal{Q}_{B}\right)$-tensors with a sequence of $H^{1}\left(\Omega, \mathcal{Q}_{B}\right)$-tensors in the strong $L^{2}$-topology. We can always assume that such a sequence exists (this follows by convolution and the convexity property of $\mathcal{Q}_{B}$ ). The discussion of the case $X=U$ is postponed until section 2.2.1.

The Gamma-convergence result holds also if we remove the constraint on the divergence of $\mathbf{u}$, as described in the next corollary.

Corollary 3. Let $\Omega \subset \mathbb{R}^{3}$ be an open, bounded, connected set with Lipschitz boundary, and let $\Gamma_{D} \subseteq \partial \Omega$ be an open subset with positive surface measure. Take $\mathbf{g}(x) \in H^{1}\left(\Omega, \mathbb{R}^{3}\right)$ and assume $f_{\text {mec }}$ as in (1.20). Define

$$
\mathrm{F}_{X, \varepsilon}(\mathbf{Q}, \mathbf{u}):=\left\{\begin{array}{cc}
\int_{\Omega}\left(\varepsilon^{2}|\nabla \mathbf{Q}|^{2}+f_{m e c}(\mathbf{Q}, \nabla \mathbf{u})\right) d x & \text { on } H^{1}\left(\Omega, \mathcal{Q}_{X}\right) \times H^{1}\left(\Omega, \mathbb{R}^{3}\right), \\
+\infty & \text { otherwise in } L^{2}\left(\Omega, \mathbb{M}^{3 \times 3}\right) \times H^{1}\left(\Omega, \mathbb{R}^{3}\right)
\end{array}\right.
$$

and

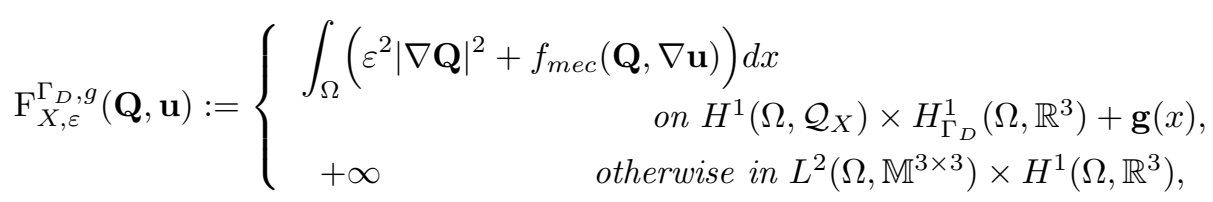

where $X$ stands for either $F r$, or $U$, or $B$. Then

$$
\Gamma(\sigma)-\lim _{\varepsilon \rightarrow 0} \mathrm{~F}_{X, \varepsilon}(\mathbf{Q}, \mathbf{u})=\mathrm{F}_{B, m e c}(\mathbf{Q}, \mathbf{u})
$$

and

$$
\Gamma(\sigma)-\lim _{\varepsilon \rightarrow 0} \mathrm{~F}_{X, \varepsilon}^{\Gamma_{D}, g}(\mathbf{Q}, \mathbf{u})=\mathrm{F}_{B, m e c}^{\Gamma_{D}, g}(\mathbf{Q}, \mathbf{u}),
$$

where $\mathrm{F}_{B, \text { mec }}$ and $\mathrm{F}_{B, \text { mec }}^{\Gamma_{D}, g}$ are defined in Theorem 1 and Corollary 1.

Proof. We refer to the proof of Theorem 3, where an analogous case is treated. We observe that it is not necessary to take sequences of displacements with divergence equal to zero.

2.2.1. Uniaxial models. We show how to obtain all the relaxation and Gammaconvergence results claimed in the theorems and corollaries above for the uniaxial model $X=U$. Consider the functionals defined in Theorem 1 under the hypotheses specified therein. In view of the inclusion $\mathcal{Q}_{F r} \subset \mathcal{Q}_{U} \subset \mathcal{Q}_{B}$, we have

$$
\mathrm{F}_{B, m e c} \leq \mathrm{F}_{U, m e c} \leq \mathrm{F}_{F r, m e c}
$$

and, by taking the relaxation,

$$
\mathrm{F}_{B, m e c}=\overline{\mathrm{F}}_{B, m e c} \leq \overline{\mathrm{F}}_{U, m e c} \leq \overline{\mathrm{F}}_{F r, m e c}=\overline{\mathrm{F}}_{B, m e c} .
$$

This proves that the relaxation of $\mathrm{F}_{U, m e c}$ is $\mathrm{F}_{B, m e c}$. Analogously, if we consider the functionals introduced in Theorem 2 and Corollaries 1 and 2, we obtain $\overline{\mathcal{F}}_{U, \text { mec }}=$ 
$\mathcal{F}_{B, \text { mec }}, \overline{\mathrm{F}}_{U, \text { mec }}^{\Gamma_{D}, g}=\mathrm{F}_{B, \text { mec }}^{\Gamma_{D}, g}$, and $\overline{\mathcal{F}}_{U, \text { mec }}^{\Gamma_{D}, g}=\mathcal{F}_{B, \text { mec }}^{\Gamma_{D}, g}$, respectively. The same chain of inequalities holds for the Gamma-limits of Theorem 3:

$$
\mathcal{F}_{B, \text { mec }}=\Gamma-\lim _{\varepsilon \rightarrow 0} \mathcal{F}_{B, \varepsilon} \leq \Gamma-\liminf _{\varepsilon \rightarrow 0} \mathcal{F}_{U, \varepsilon} \leq \Gamma-\limsup _{\varepsilon \rightarrow 0} \mathcal{F}_{U, \varepsilon} \leq \Gamma-\limsup _{\varepsilon \rightarrow 0} \mathcal{F}_{F r, \varepsilon}=\mathcal{F}_{B, \text { mec }},
$$

yielding $\Gamma-\lim _{\varepsilon \rightarrow 0} \mathcal{F}_{U, \varepsilon}=\mathcal{F}_{B, \text { mec }}$ and, similarly, $\Gamma-\lim _{\varepsilon \rightarrow 0} \mathcal{F}_{U, \varepsilon}^{\Gamma_{D}, g}=\mathcal{F}_{B, \text { mec }}^{\Gamma_{D}, g}$. Then the Gamma-convergence results for the functionals defined in Corollary 3 in the case $X=U$ follow similarly.

2.3. Discussion. In this section we apply our relaxation and Gamma-convergence results to some concrete examples, and we discuss some physical implications of our analysis.

Physical interpretation. In the case in which we model the order of the system according to the Frank theory, we accept a direct coupling between strain and local orientation of the liquid crystal molecules. Experimental results show that a uniaxial stretch aligns the molecules along the direction of maximal stretch. Whether a macroscopic deformation may alter also the local order of the molecules and not only the local direction is a debated problem. Therefore, the direct coupling imposed by (1.1) may be accepted if $\mathbf{Q} \in \mathcal{Q}_{F r}$, while it may seem too simplistic if $\mathbf{Q} \in \mathcal{Q}_{U}$ or $\mathcal{Q}_{B}$. We reveal the main consequences of our relaxation and Gamma-convergence results.

COROLlary 4. Under the hypotheses of Theorem 2 and Corollary 2

$$
\inf _{L^{2}\left(\Omega, \mathbb{M}^{3 \times 3}\right) \times H^{1}\left(\Omega, \mathbb{R}^{3}\right)} \mathcal{F}_{X, m e c}^{\Gamma_{D}, g}(\mathbf{Q}, \mathbf{u})=\min _{L^{2}\left(\Omega, \mathbb{M}^{3} \times 3\right) \times H^{1}\left(\Omega, \mathbb{R}^{3}\right)} \mathcal{F}_{B, m e c}^{\Gamma_{D}, g}(\mathbf{Q}, \mathbf{u}) .
$$

Moreover, let $\left\{\mathbf{Q}_{j}, \mathbf{u}_{j}\right\}$ be a minimizing sequence of $\mathcal{F}_{X, \varepsilon_{j}}^{\Gamma_{D}, g}$. Then there exists a subsequence which converges to a solution of the right-hand side of (2.57) with respect to the topology $\sigma$. Finally, we have

$$
\lim _{j \rightarrow+\infty}\left[\inf \mathcal{F}_{X, \varepsilon_{j}}^{\Gamma_{D}, g}\right]=\min \mathcal{F}_{B, m e c}^{\Gamma_{D}, g} .
$$

Proof. Equation (2.57) follows from a well-known property of the relaxation (see [14]). For the reader's convenience, we show that the minimum on the right-hand side of $(2.57)$ is attained. Since the function $(\mathbf{Q}, \mathbf{F}) \mapsto \mu|\mathbb{E}(\mathbf{F})-\gamma \mathbf{Q}|^{2}+\frac{\lambda}{2}(\operatorname{tr} \mathbf{F})^{2}$ is convex, $\mathcal{F}_{B, \text { mec }}^{\Gamma_{D}, g}$ is weakly lower semicontinuous. Moreover, the $H^{1}$-norm of the displacement $\mathbf{u}$ is controlled by Korn's inequality (1.12) and Poincaré's inequality [10, Theorem 6.1-8] thanks to the coercivity condition (1.21), and the $L^{2}$-norm of $\mathbf{Q}$ is bounded because $\mathcal{Q}_{B}$ is compact. The second part of Corollary 4 is a consequence of the fundamental theorem of Gamma-convergence (see [4], [14]).

Remark 6. A result analogous to that of Corollary 4 holds also for the energies of compressible elastomers of Theorem 1 and Corollary 1.

Corollary 4 has a relevant physical interpretation. The equilibrium solution to the left-hand side of problem (2.57) is characterized by a biaxial tensor field. This is true not only if the elastomer is modeled in the frame of the de Gennes theory, but also in the case of the Frank tensor by developing an effective biaxial microstructure. Interestingly, we obtain the full information associated with the biaxial order tensor theory, that is, the possibility of describing isotropy and low order phases, thus justifying the material's instabilities observed experimentally [3], [29]. 
Relaxation of the macroscopic model. The asymptotic analysis presented in section 2 is not the only possible way to model formation of microstructure in nematic elastomers. We show that there exists a relation between the Gamma-convergence result of Theorem 2 and the relaxation of another nonconvex energy for nematic elastomers presented in [7]. Furthermore, as a by-product of this discussion, we solve explicitly the minimization problem (2.57) in the presence of an affine traction imposed on the boundary of the domain.

We define

$$
f_{X}(\mathbf{F}):=\inf _{\mathbf{Q} \in \mathcal{Q}_{X}} f_{m e c}(\mathbf{Q}, \mathbf{F})=\inf _{\mathbf{Q} \in \mathcal{Q}_{X}} \mu|\mathbb{E}(\mathbf{F})-\gamma \mathbf{Q}|^{2}+\frac{\lambda}{2}(\operatorname{tr} \mathbf{F})^{2},
$$

where $X$ stands for either $F r$, or $U$, or $B$. Clearly, the new functions obtained in (2.59) are the measure of the distance from the set $\gamma \mathcal{Q}_{X}$ :

$$
\inf _{\mathbf{Q} \in \mathcal{Q}_{X}} \mu|\mathbb{E}(\mathbf{F})-\gamma \mathbf{Q}|^{2}+\frac{\lambda}{2}(\operatorname{tr} \mathbf{F})^{2}=\mu \operatorname{dist}^{2}\left(\mathbb{E}(\mathbf{F}), \gamma \mathcal{Q}_{X}\right)+\frac{\lambda}{2}(\operatorname{tr} \mathbf{F})^{2} .
$$

In this new model the influence of the internal nematic variable $\mathbf{Q}$ is perceived only through its coupling to the strain. In fact, the interaction between the macroscopic variable $\mathbb{E}(\mathbf{F})$ and the microscopic variable $\mathbf{Q}$ is solved by substituting in $\mathbf{Q} \rightarrow f_{m e c}(\cdot, \mathbf{F})$ the optimal tensor field generated by a constant strain. Hence, we obtain a macroscopic model ruled by the displacement. The explicit expressions of $f_{F r}, f_{U}$, and $f_{B}$ are reported in [8] and [7]. We observe that, since $\mathcal{Q}_{F r} \subset \mathcal{Q}_{U} \subset \mathcal{Q}_{B}$, it follows that $f_{B}(\mathbf{F}) \leq f_{U}(\mathbf{F}) \leq f_{F r}(\mathbf{F})$. Moreover, it turns out that $f_{F r}$ and $f_{U}$ are nonconvex energy densities, while $f_{B}$ is convex. In [7] we study minimization problems for functionals defined by integrating the densities $f_{F r}, f_{U}$, and $f_{B}$ over the reference configuration $\Omega$. In the case of the biaxial theory, namely, if we assume $f_{B}$, we obtain compactness of the minimizing sequences and weak lower semicontinuity by convexity. On the other hand, the functionals which we define in the cases $X=F r$ or $X=U$ are not weakly lower semicontinuous, and we prove that their relaxation is precisely the energy defined for $X=B$. Hence, also in the scenario of the macroscopic models, we obtain an effective de Gennes-type microstructure via relaxation. This fact suggests investigating possible connections between the Gamma-limits of section 2 and the relaxation results of [7]. We recall the main theorem regarding the macroscopic models.

Theorem 4 (see [7, Theorems 1 and 2]). Let $\Omega \subset \mathbb{R}^{3}$ be an open, bounded, connected set with Lipschitz boundary, and let $\Gamma_{D} \subseteq \partial \Omega$ be an open subset with positive surface measure. Assume $f_{X}(\cdot)$ as in $(2.59)$. Take $\varphi(x)$ and $\mathbf{g}(x)$ in $H^{1}\left(\Omega, \mathbb{R}^{3}\right)$ with $\operatorname{div} \mathbf{g}(x)=0$ a.e. in $\Omega$ and define in $H^{1}\left(\Omega, \mathbb{R}^{3}\right)$

$$
\mathrm{J}_{X}(\mathbf{u}):=\int_{\Omega} f_{X}(\nabla \mathbf{u}) d x \quad \text { and } \quad \mathrm{J}_{X}^{\Gamma_{D}, \varphi}(\mathbf{u}):=\left\{\begin{array}{cc}
\mathrm{J}_{X}(\mathbf{u}) & \text { on } H_{\Gamma_{D}}^{1}\left(\Omega, \mathbb{R}^{3}\right)+\varphi(x), \\
+\infty & \text { otherwise }
\end{array}\right.
$$

and

$\mathcal{J}_{X}(\mathbf{u}):=\left\{\begin{array}{cc}\mathrm{J}_{X}(\mathbf{u}) & \text { if } \operatorname{div} \mathbf{u}=0, \\ +\infty & \text { otherwise, }\end{array} \quad \mathcal{J}_{X}^{\Gamma_{D}, g}(\mathbf{u}):=\left\{\begin{array}{c}\mathrm{J}_{X}(\mathbf{u}) \text { on } H_{\Gamma_{D}}^{1}+\mathbf{g}(x), \operatorname{div} \mathbf{u}=0, \\ +\infty\end{array}\right.\right.$

where $X$ stands for either $F r$, or $U$, or $B$. Then the relaxation of $\mathrm{J}_{X}, \mathrm{~J}_{X}^{\Gamma_{D}, \varphi}, \mathcal{J}_{X}$, $\mathcal{J}_{X}^{\Gamma_{D}, g}$ is, respectively, $\mathrm{J}_{B}, \mathrm{~J}_{B}^{\Gamma_{D}, \varphi}, \mathcal{J}_{B}, \mathcal{J}_{B}^{\Gamma_{D}, g}$. Furthermore, $f_{B}$ satisfies a solenoidal quasi-convexification formula, namely,

$$
f_{B}(\mathbf{Z})=\inf \left\{|\omega|^{-1} \int_{\omega} f_{X}(\mathbf{Z}+\nabla \mathbf{w}(y)) d y: \mathbf{w} \in H_{o}^{1}\left(\omega, \mathbb{R}^{3}\right), \operatorname{div} \mathbf{w}=0\right\}
$$


$\forall \mathbf{Z} \in \mathbb{M}_{0}^{3 \times 3}$, where $\omega$ is any nonempty, open, bounded, connected subset of $\mathbb{R}^{3}$ with Lipschitz boundary.

In order to compare minima and minimizers of $\mathcal{F}_{B, \text { mec }}^{\Gamma_{D}, g}$ and of $\mathcal{J}_{B}^{\Gamma_{D}, g}$, we start by manipulating (2.57):

$$
\begin{aligned}
& \min _{\substack{L^{2}\left(\Omega, \mathbb{M}^{3 \times 3}\right) \\
\times H^{1}\left(\Omega, \mathbb{R}^{3}\right)}} \mathcal{F}_{B, m e c}^{\Gamma_{D}, g}(\mathbf{Q}, \mathbf{u})=\inf _{\substack{\mathbf{u} \in H_{\Gamma_{D}}^{1}\left(\Omega, \mathbb{R}^{3}\right)+\mathbf{g}(x), \operatorname{div} \mathbf{u}=0}} \min _{\mathbf{Q} \in L^{2}\left(\Omega, \mathcal{Q}_{B}\right)} \int_{\Omega} \mu|\mathbb{E}(\nabla \mathbf{u})-\gamma \mathbf{Q}|^{2} d x \\
& =\inf _{\mathbf{u} \in H_{\Gamma_{D}}^{1}\left(\Omega, \mathbb{R}^{3}\right)+\mathbf{g}(x),} \mu \operatorname{dist}_{L^{2}}^{2}\left(\mathbb{E}(\nabla \mathbf{u}), \gamma L^{2}\left(\Omega, \mathcal{Q}_{B}\right)\right) .
\end{aligned}
$$

Thanks to Proposition 4 and (1.17), we can write

$$
\mu \operatorname{dist}_{L^{2}}^{2}\left(\mathbb{E}(\nabla \mathbf{u}), L^{2}\left(\Omega, \gamma \mathcal{Q}_{B}\right)\right)=\mu \int_{\Omega} \operatorname{dist}^{2}\left(\mathbb{E}(\nabla \mathbf{u}(x)), \gamma \mathcal{Q}_{B}\right) d x
$$

and, summarizing,

$$
\min _{L^{2}\left(\Omega, \mathbb{M}^{3 \times 3}\right) \times H^{1}\left(\Omega, \mathbb{R}^{3}\right)} \mathcal{F}_{B, m e c}^{\Gamma_{D}, g}(\mathbf{Q}, \mathbf{u})=\min _{H^{1}\left(\Omega, \mathbb{R}^{3}\right)} \mathcal{J}_{B}^{\Gamma_{D}, g}(\mathbf{u}) .
$$

Therefore, at equilibrium, the energy is the same in both models. We continue our discussion in order to obtain information on minimizers. Considering again (2.63), we label with $(\overline{\mathbf{Q}}, \overline{\mathbf{u}})$ a minimizer of $\mathcal{F}_{B, \text { mec }}^{\Gamma_{D}, g}$ and with $\overline{\overline{\mathbf{u}}}$ a minimizer of $\mathcal{J}_{B}^{\Gamma_{D}, g}$. Hence, if we define $\overline{\overline{\mathbf{Q}}}:=\pi^{\mathcal{Q}_{B}}(\mathbb{E}(\nabla \overline{\overline{\mathbf{u}}}) / \gamma)$, we can write

$$
\mu\|\mathbb{E}(\nabla \overline{\mathbf{u}})-\gamma \overline{\mathbf{Q}}\|_{L^{2}\left(\Omega, \mathbb{M}^{3 \times 3}\right)}^{2}=\mathcal{F}_{B, m e c}^{\Gamma_{D}, g}(\overline{\mathbf{Q}}, \overline{\mathbf{u}})=\mathcal{J}_{B}^{\Gamma_{D}, g}(\overline{\overline{\mathbf{u}}})=\mu \int_{\Omega}|\mathbb{E}(\nabla \overline{\overline{\mathbf{u}}})-\gamma \overline{\overline{\mathbf{Q}}}|^{2} d x
$$

This is not sufficient, of course, to conclude that $(\overline{\mathbf{Q}}, \overline{\mathbf{u}})$ coincides with $(\overline{\overline{\mathbf{Q}}}, \overline{\overline{\mathbf{u}}})$, which would be true, for instance, if both $\mathcal{F}_{B, \text { mec }}^{\Gamma_{D}, g}$ and $\mathcal{J}_{B}^{\Gamma_{D}, g}$ were strictly convex. Anyway, it is clear that $(\overline{\mathbf{Q}}, \overline{\mathbf{u}})$ minimizes $\mathcal{J}_{B}^{\Gamma_{D}, g}$ and that $(\overline{\overline{\mathbf{Q}}}, \overline{\overline{\mathbf{u}}})$ minimizes $\mathcal{F}_{B, \text { mec }}^{\Gamma_{D}, g}$ as well. This simple observation suggests that, in order to find a minimizer of $\mathcal{F}_{B, \text { mec }}^{\Gamma_{D}, g}$, one can solve the minimization problem for $\mathcal{J}_{B}^{\Gamma_{D}, g}$, which, at least in some cases, may be more convenient. As an example, we turn to the relevant situation where $\Gamma_{D}=\partial \Omega$ and $\mathbf{g}(x)=\mathbf{F} x$, with $\mathbf{F} \in \mathbb{M}_{0}^{3 \times 3}$. Thanks to (2.60) (in the trivial case $X=B$ ), we have

$$
\int_{\Omega} f_{B}(\mathbf{F}) d x \leq \int_{\Omega} f_{B}(\mathbf{F}+\nabla \mathbf{w}) d x \quad \forall \mathbf{w} \in H_{o}^{1}\left(\Omega, \mathbb{R}^{3}\right) \text { with } \operatorname{div} \mathbf{w}=0
$$

and hence a minimizer of $\mathcal{F}_{B, \text { mec }}^{\Gamma_{D}, g}$ is

$$
(\overline{\mathbf{Q}}, \overline{\mathbf{u}})=\left(\pi^{\mathcal{Q}_{B}}(\mathbb{E}(\mathbf{F}) / \gamma), \mathbf{F} x\right) .
$$

To conclude, we observe that an analogous discussion holds for minima and minimizers of the functionals for compressible elastomers $\mathrm{F}_{B, m e c}^{\Gamma_{D}, g}$ and $\mathrm{J}_{B}^{\Gamma_{D}, g}$.

Copyright (c) by SIAM. Unauthorized reproduction of this article is prohibited. 
3. Small particles: Asymptotics. We turn to the asymptotic analysis for small particles. The space $L^{2}\left(\Omega, \mathbb{M}^{3 \times 3}\right) \times H^{1}\left(\Omega, \mathbb{R}^{3}\right)$ is now endowed with the strong topology of $L^{2}\left(\Omega, \mathbb{M}^{3 \times 3}\right)$ for the variable $\mathbf{Q}$ and the weak topology of $H^{1}\left(\Omega, \mathbb{R}^{3}\right)$ for $\mathbf{u}$, and we write

$$
\sigma^{\prime}:=\text { strong- } L^{2}\left(\Omega, \mathbb{M}^{3 \times 3}\right) \times \text { weak- } H^{1}\left(\Omega, \mathbb{R}^{3}\right) .
$$

In this scenario, any relaxation phenomenon is ruled out and, as a consequence, the analysis turns out to be simplified. In what follows, we denote by

$$
\left\{H^{1}\left(\Omega, \mathcal{Q}_{X}\right), \text { Const }\right\}:=\left\{\mathbf{Q}: \Omega \rightarrow \mathcal{Q}_{X}, \text { Const }\right\},
$$

where $X$ stands for either $F r$, or $U$, or $B$, the subset in $H^{1}\left(\Omega, \mathcal{Q}_{X}\right)$ of the constant tensors. Observe that the weak (and strong) topology of $H^{1}\left(\Omega, \mathbb{M}^{3 \times 3}\right)$ on $\left\{H^{1}\left(\Omega, \mathbb{M}^{3 \times 3}\right)\right.$, Const $\}$ coincide with the standard topology of $\mathbb{M}^{3 \times 3}$.

3.1. Gamma-convergence theorem. We state the Gamma-convergence result for incompressible materials.

THEOREM 5. Let $\Omega \subset \mathbb{R}^{3}$ be an open, bounded, connected set with Lipschitz boundary, and let $\Gamma_{D} \subseteq \partial \Omega$ be an open subset with positive surface measure. Take $\mathbf{g}(x) \in H^{1}\left(\Omega, \mathbb{R}^{3}\right)$ with $\operatorname{div} \mathbf{g}=0$. Assume $f_{\text {mec }}$ as in $(1.20)$, and $\mathcal{F}_{X, \varepsilon}$ and $\mathcal{F}_{X, \varepsilon}^{\Gamma_{D}, g}$ as in Theorem 3, that is,

$$
\mathcal{F}_{X, \varepsilon}(\mathbf{Q}, \mathbf{u})= \begin{cases}\int_{\Omega}\left(\varepsilon^{2}|\nabla \mathbf{Q}|^{2}+f_{\text {mec }}(\mathbf{Q}, \nabla \mathbf{u})\right) d x \\
+\infty \quad \begin{array}{l}
\text { on } H^{1}\left(\Omega, \mathcal{Q}_{X}\right) \times H^{1}\left(\Omega, \mathbb{R}^{3}\right), \operatorname{div} \mathbf{u}=0, \\
\text { otherwise in } L^{2}\left(\Omega, \mathbb{M}^{3 \times 3}\right) \times H^{1}\left(\Omega, \mathbb{R}^{3}\right)
\end{array}\end{cases}
$$

and

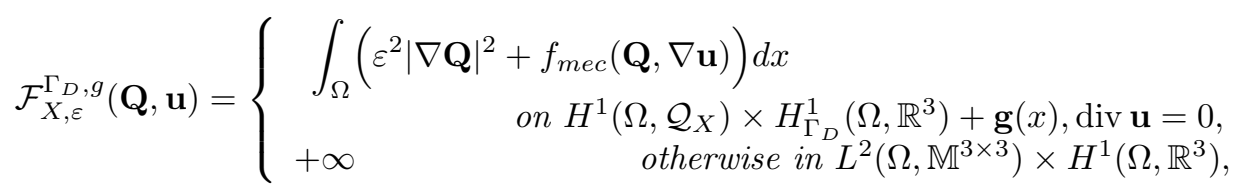

where $X$ stands for either $F r$, or $U$, or B. Then

$$
\Gamma\left(\sigma^{\prime}\right)-\lim _{\varepsilon \rightarrow+\infty} \mathcal{F}_{X, \varepsilon}(\mathbf{Q}, \mathbf{u})=\mathcal{G}_{X, m e c}(\mathbf{Q}, \mathbf{u})
$$

and

$$
\Gamma\left(\sigma^{\prime}\right)-\lim _{\varepsilon \rightarrow+\infty} \mathcal{F}_{X, \varepsilon}^{\Gamma_{D}, g}(\mathbf{Q}, \mathbf{u})=\mathcal{G}_{X, m e c}^{\Gamma_{D}, g}(\mathbf{Q}, \mathbf{u})
$$

where

$$
\mathcal{G}_{X, m e c}(\mathbf{Q}, \mathbf{u}):=\left\{\begin{array}{rr}
\int_{\Omega} f_{\text {mec }}(\mathbf{Q}, \nabla \mathbf{u}) d x \\
+\infty & \text { on }\left\{H^{1}\left(\Omega, \mathcal{Q}_{X}\right), \text { Const }\right\} \times H^{1}\left(\Omega, \mathbb{R}^{3}\right), \operatorname{div} \mathbf{u}=0, \\
+ \text { otherwise in } L^{2}\left(\Omega, \mathbb{M}^{3 \times 3}\right) \times H^{1}\left(\Omega, \mathbb{R}^{3}\right)
\end{array}\right.
$$

and

$$
\mathcal{G}_{X, m e c}^{\Gamma_{D}, g}(\mathbf{Q}, \mathbf{u}):=\left\{\begin{array}{r}
\int_{\Omega} f_{m e c}(\mathbf{Q}, \nabla \mathbf{u}) d x \\
\text { on }\left\{H^{1}\left(\Omega, \mathcal{Q}_{X}\right), \text { Const }\right\} \times H_{\Gamma_{D}}^{1}\left(\Omega, \mathbb{R}^{3}\right)+\mathbf{g}, \operatorname{div} \mathbf{u}=0, \\
+\infty \begin{array}{l}
\text { otherwise in } L^{2}\left(\Omega, \mathbb{M}^{3 \times 3}\right) \times H^{1}\left(\Omega, \mathbb{R}^{3}\right) .
\end{array}
\end{array}\right.
$$


Proof. Consider first (3.4). As $\varepsilon \rightarrow+\infty$, we extract a countable subsequence $\left\{\varepsilon_{j}\right\}$ such that $\varepsilon_{j} \rightarrow+\infty$ as $j \rightarrow+\infty$. We characterize again the Gamma-limit by the liminf and limsup inequalities.

Liminf inequality. It is enough to show that, for any $\left\{\mathbf{Q}_{j}, \mathbf{u}_{j}\right\} \subset L^{2}\left(\Omega, \mathcal{Q}_{B}\right) \times$ $H^{1}\left(\Omega, \mathbb{R}^{3}\right)$ such that

$$
\mathbf{Q}_{j}, \mathbf{u}_{j} \stackrel{\sigma^{\prime}}{\rightarrow} \mathbf{Q}, \mathbf{u} \quad \text { as } j \rightarrow+\infty
$$

we have

$$
\mathcal{G}_{X, m e c}^{\Gamma_{D}, g}(\mathbf{Q}, \mathbf{u}) \leq \liminf _{j \rightarrow+\infty} \mathcal{F}_{X, \varepsilon_{j}}^{\Gamma_{D}, g}\left(\mathbf{Q}_{j}, \mathbf{u}_{j}\right)
$$

If we pass to subsequences (here not relabeled) for which the right-hand side of (3.6) is uniformly bounded by some constant $C$, we obtain

$$
\begin{aligned}
& \liminf _{j \rightarrow+\infty} \int_{\Omega}\left|\mathbb{E}\left(\nabla \mathbf{u}_{j}\right)-\gamma \mathbf{Q}_{j}\right|^{2} d x \\
& \quad \leq \liminf _{j \rightarrow+\infty} \int_{\Omega}\left(\varepsilon_{j}^{2}\left|\nabla \mathbf{Q}_{j}\right|^{2}+\left|\mathbb{E}\left(\nabla \mathbf{u}_{j}\right)-\gamma \mathbf{Q}_{j}\right|^{2}\right) d x \leq C .
\end{aligned}
$$

Moreover, observe that

$$
\int_{\Omega} \varepsilon_{j}^{2}\left|\nabla \mathbf{Q}_{j}\right|^{2} d x \leq \mathcal{F}_{X, \varepsilon_{j}}^{\Gamma_{D}, g}\left(\mathbf{Q}_{j}, \mathbf{u}_{j}\right) \leq C,
$$

and hence $\int_{\Omega}\left|\nabla \mathbf{Q}_{j}\right|^{2} d x \rightarrow 0$ as $j \rightarrow+\infty$. Therefore, by Korn's and Poincaré's inequalities and the properties of the trace, it follows that the set where the functional is finite is

$$
\left\{H^{1}\left(\Omega, \mathcal{Q}_{X}\right), \text { Const }\right\} \times\left\{H_{\Gamma_{D}}^{1}\left(\Omega, \mathbb{R}^{3}\right)+\mathbf{g}(x), \operatorname{div} \mathbf{u}=0\right\} .
$$

The claim follows trivially from (3.7).

Limsup inequality. Given any $(\mathbf{Q}, \mathbf{u}) \in L^{2}\left(\Omega, \mathbb{M}^{3 \times 3}\right) \times H^{1}\left(\Omega, \mathbb{R}^{3}\right)$ we have to exhibit a sequence $\left\{\widehat{\mathbf{Q}}_{j}, \widehat{\mathbf{u}}_{j}\right\} \subset L^{2}\left(\Omega, \mathbb{M}^{3 \times 3}\right) \times H^{1}\left(\Omega, \mathbb{R}^{3}\right)$ such that

$$
\widehat{\mathbf{Q}}_{j} \rightarrow \mathbf{Q} \text { in } L^{2}\left(\Omega, \mathbb{M}^{3 \times 3}\right), \quad \widehat{\mathbf{u}}_{j} \rightarrow \mathbf{u} \text { in } H^{1}\left(\Omega, \mathbb{R}^{3}\right) \text { as } j \rightarrow+\infty,
$$

yielding

$$
\mathcal{G}_{X, m e c}^{\Gamma_{D}, g}(\mathbf{Q}, \mathbf{u})=\limsup _{j \rightarrow+\infty} \mathcal{F}_{X, \varepsilon_{j}}^{\Gamma_{D}, g}\left(\widehat{\mathbf{Q}}_{j}, \widehat{\mathbf{u}}_{j}\right)
$$

To obtain the claim it is sufficient to take the trivial sequence $\left\{\widehat{\mathbf{Q}}_{j}, \widehat{\mathbf{u}}_{j}\right\} \equiv(\mathbf{Q}, \mathbf{u})$. The proof of (3.3) is similar.

In the following corollary we present the Gamma-convergence result for small samples of compressible elastomers.

Corollary 5. Let $\Omega \subset \mathbb{R}^{3}$ be an open, bounded, connected set with Lipschitz boundary, and let $\Gamma_{D} \subseteq \partial \Omega$ be an open subset with positive surface measure. Take $\mathbf{g}(x) \in H^{1}\left(\Omega, \mathbb{R}^{3}\right)$. Assume $f_{m e c}$ as in $(1.20)$, and $F_{X, \varepsilon}$ and $F_{X, \varepsilon}^{\Gamma_{D}, g}$ as in Corollary 3 , that is,

$$
\mathrm{F}_{X, \varepsilon}(\mathbf{Q}, \mathbf{u}):=\left\{\begin{array}{cr}
\int_{\Omega}\left(\varepsilon^{2}|\nabla \mathbf{Q}|^{2}+f_{m e c}(\mathbf{Q}, \nabla \mathbf{u})\right) d x & \text { on } H^{1}\left(\Omega, \mathcal{Q}_{X}\right) \times H^{1}\left(\Omega, \mathbb{R}^{3}\right), \\
+\infty & \text { otherwise in } L^{2}\left(\Omega, \mathbb{M}^{3 \times 3}\right) \times H^{1}\left(\Omega, \mathbb{R}^{3}\right)
\end{array}\right.
$$

Copyright $@$ by SIAM. Unauthorized reproduction of this article is prohibited. 
and

$$
\mathrm{F}_{X, \varepsilon}^{\Gamma_{D}, g}(\mathbf{Q}, \mathbf{u}):=\left\{\begin{array}{cr}
\int_{\Omega}\left(\varepsilon^{2}|\nabla \mathbf{Q}|^{2}+f_{m e c}(\mathbf{Q}, \nabla \mathbf{u})\right) d x \\
+\infty & \text { on } H^{1}\left(\Omega, \mathcal{Q}_{X}\right) \times H_{\Gamma_{D}}^{1}\left(\Omega, \mathbb{R}^{3}\right)+\mathbf{g}(x), \\
+\infty & \text { otherwise in } L^{2}\left(\Omega, \mathbb{M}^{3 \times 3}\right) \times H^{1}\left(\Omega, \mathbb{R}^{3}\right),
\end{array}\right.
$$

where $X$ stands for either $F r$, or $U$, or $B$. Then

$$
\Gamma\left(\sigma^{\prime}\right)-\lim _{j \rightarrow+\infty} \mathrm{F}_{X, \varepsilon}(\mathbf{Q}, \mathbf{u})=\mathrm{G}_{X, m e c}(\mathbf{Q}, \mathbf{u})
$$

and

$$
\Gamma\left(\sigma^{\prime}\right)-\lim _{j \rightarrow+\infty} \mathrm{F}_{X, \varepsilon}^{\Gamma_{D}, g}(\mathbf{Q}, \mathbf{u})=\mathrm{G}_{X, m e c}^{\Gamma_{D}, g}(\mathbf{Q}, \mathbf{u})
$$

where

$$
\mathrm{G}_{X, m e c}(\mathbf{Q}, \mathbf{u}):=\left\{\begin{array}{cc}
\int_{\Omega} f_{m e c}(\mathbf{Q}, \nabla \mathbf{u}) d x & \text { on }\left\{H^{1}\left(\Omega, \mathcal{Q}_{X}\right), \text { Const }\right\} \times H^{1}\left(\Omega, \mathbb{R}^{3}\right) \\
+\infty & \text { otherwise in } L^{2}\left(\Omega, \mathbb{M}^{3 \times 3}\right) \times H^{1}\left(\Omega, \mathbb{R}^{3}\right)
\end{array}\right.
$$

and

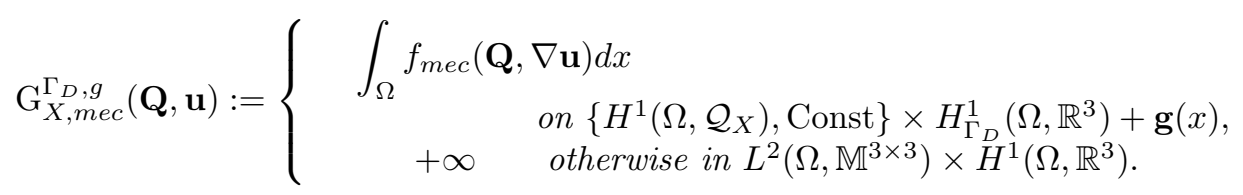

Proof. We refer to the proof of Theorem 5. Note that it is not necessary to take sequences of displacements with divergence equal to zero.

3.2. Discussion. It is easy to show that the problem

$$
\min _{L^{2}\left(\Omega, \mathbb{M}^{3 \times 3}\right) \times H^{1}\left(\Omega, \mathbb{R}^{3}\right)} \mathcal{G}_{X, \text { mec }}^{\Gamma_{D}, g}(\mathbf{Q}, \mathbf{u})
$$

has at least one solution. The coercivity condition (1.21) and the compactness of $\mathcal{Q}_{X}$ guarantee that we have boundedness of minimizing sequences. Then the weak lower semicontinuity of $\mathcal{G}_{X, \text { mec }}^{\Gamma_{D}, g}$ follows trivially.

Problem (3.14) has a very relevant role in the engineering literature, since minimizers of $\mathcal{G}_{X, \text { mec }}^{\Gamma_{D}, g}$ capture the asymptotic behavior of minimizers of $\mathcal{F}_{X, \varepsilon}^{\Gamma_{D}, g}$ in the following sense. Let $\left\{\mathbf{Q}_{j}, \mathbf{u}_{j}\right\}$ be a minimizer of $\mathcal{F}_{X, \varepsilon_{j}}^{\Gamma_{D}, g}$ and let $(\mathbf{Q}, \mathbf{u})$ be a minimizer of $\mathcal{G}_{X, m e c}^{\Gamma_{D}, g}$. Then

$$
\mathcal{F}_{X, \varepsilon_{j}}^{\Gamma_{D}, g}\left(\mathbf{Q}_{j}, \mathbf{u}_{j}\right) \rightarrow \mathcal{G}_{X, m e c}^{\Gamma_{D}, g}(\mathbf{Q}, \mathbf{u}) \text { as } j \rightarrow+\infty
$$

and there exists a subsequence of $\left\{\mathbf{Q}_{j}, \mathbf{u}_{j}\right\}$ which converges to a solution of (3.14) with respect to the topology $\sigma^{\prime}$. Interestingly, in the case in which we impose an affine boundary conditions for the displacement $\mathbf{u}$, it is possible to solve (3.14) explicitly. To this end, observe that it is possible to write

$$
\begin{aligned}
& \min _{L^{2}\left(\Omega, \mathbb{M}^{3 \times 3}\right) \times H^{1}\left(\Omega, \mathbb{R}^{3}\right)} \mathcal{G}_{X, m e c}^{\Gamma_{D}, g}(\mathbf{Q}, \mathbf{u}) \\
& =\inf _{\left\{\mathbf{Q} \in H^{1}\left(\Omega, \mathcal{Q}_{X}\right), \text { Const }\right\}} \min _{\mathbf{u} \in H_{\Gamma_{D}}^{1}\left(\Omega, \mathbb{R}^{3}\right)+\mathbf{g}, \operatorname{div} \mathbf{u}=0} \int_{\Omega} \mu|\mathbb{E}(\nabla \mathbf{u})-\gamma \mathbf{Q}|^{2} d x .
\end{aligned}
$$

Copyright $@$ by SIAM. Unauthorized reproduction of this article is prohibited. 
In view of Korn's inequality (1.12) and by the strict convexity of the function $\mathbf{E} \mapsto$ $|\cdot-\gamma \mathbf{Q}|^{2}$, the minimization problem

$$
\min _{\mathbf{u} \in H_{\Gamma_{D}}^{1}\left(\Omega, \mathbb{R}^{3}\right)+\mathbf{g}, \operatorname{div} \mathbf{u}=0} \int_{\Omega} \mu|\mathbb{E}(\nabla \mathbf{u})-\gamma \mathbf{Q}|^{2} d x
$$

has a unique solution which we denote by $\overline{\mathbf{u}}$. Now, we label with $\overline{\mathbf{E}}^{a v} \in \mathbb{M}_{0}^{3 \times 3}$ the average of the tensor field $\mathbb{E}(\nabla \overline{\mathbf{u}})$, namely, ${ }^{2}$

$$
\mathbf{E}^{a v}:=\frac{1}{|\Omega|} \int_{\Omega} \mathbb{E}(\nabla \overline{\mathbf{u}}) d x
$$

and define $\overline{\mathbf{E}}^{\sim}(x):=\mathbb{E}(\nabla \overline{\mathbf{u}})-\overline{\mathbf{E}}^{a v}$. Since $\overline{\mathbf{E}}^{a v}$ and $\mathbf{Q}$ are constant matrices, we have

$$
\int_{\Omega}\left(\overline{\mathbf{E}}^{a v}-\gamma \mathbf{Q}\right): \overline{\mathbf{E}}^{\sim}(x) d x=0 .
$$

Therefore, (3.16) reads

$$
\mu \int_{\Omega}\left|\left(\overline{\mathbf{E}}^{a v}-\gamma \mathbf{Q}\right)+\overline{\mathbf{E}}^{\sim}(x)\right|^{2} d x=\mu \int_{\Omega}\left(\left|\overline{\mathbf{E}}^{a v}-\gamma \mathbf{Q}\right|^{2}+\left|\overline{\mathbf{E}}^{\sim}(x)\right|^{2}\right) d x
$$

and (3.15) can be formulated as follows:

$$
\begin{aligned}
& \min _{L^{2}\left(\Omega, \mathbb{M}^{3 \times 3}\right) \times H^{1}\left(\Omega, \mathbb{R}^{3}\right)} \mathcal{G}_{X, m e c}^{\Gamma_{D}, g}(\mathbf{Q}, \mathbf{u})=\underset{\left\{\mathbf{Q} \in H^{1}\left(\Omega, \mathcal{Q}_{X}\right), \text { Const }\right\}}{\inf } \mu \int_{\Omega}\left|\overline{\mathbf{E}}^{a v}-\gamma \mathbf{Q}\right|^{2} d x \\
&+\mu \int_{\Omega}\left|\overline{\mathbf{E}}^{\sim}(x)\right|^{2} d x=\mu \int_{\Omega} \operatorname{dist}^{2}\left(\overline{\mathbf{E}}^{a v}, \gamma \mathcal{Q}_{X}\right) d x+\mu \int_{\Omega}\left|\overline{\mathbf{E}}^{\sim}(x)\right|^{2} d x .
\end{aligned}
$$

Now, let $\Gamma_{D}=\partial \Omega$ and $\mathbf{g}(x)=\mathbf{F} x$ with $\mathbf{F} \in \mathbb{M}_{0}^{3 \times 3}$. By the strict convexity of $\mathbf{E} \rightarrow|\cdot-\gamma \mathbf{Q}|^{2}$ and since $\mathbf{Q} \in \mathcal{Q}_{X}$ is constant, we can write

$$
\begin{gathered}
\int_{\Omega} \mu|\mathbb{E}(\mathbf{F})-\gamma \mathbf{Q}|^{2} d x \leq \int_{\Omega} \mu|\mathbb{E}(\mathbf{F}+\nabla \mathbf{w})-\gamma \mathbf{Q}|^{2} d x \\
\forall \mathbf{w} \in H_{o}^{1}\left(\Omega, \mathbb{R}^{3}\right) \text { with } \operatorname{div} \mathbf{w}=0,
\end{gathered}
$$

which proves that $\overline{\mathbf{u}}=\mathbf{F} x$ for every $x$ in $\bar{\Omega}$. Writing $\overline{\mathbf{E}}=\overline{\mathbf{E}}^{a v}$, then (3.20) becomes

$$
\min _{L^{2}\left(\Omega, \mathbb{M}^{3} \times 3\right) \times H^{1}\left(\Omega, \mathbb{R}^{3}\right)} \mathcal{G}_{X, m e c}^{\Gamma_{D}, g}(\mathbf{Q}, \mathbf{u})=\mu \int_{\Omega} \operatorname{dist}^{2}\left(\overline{\mathbf{E}}, \gamma \mathcal{Q}_{X}\right) d x,
$$

and the minimization problem on the left-hand side of (3.21) can now be solved algebraically by computing the element of minimum distance from $\gamma \mathcal{Q}_{X}$. We find the exact asymptotic expressions of the minimizers of the energy parameterized by the mechanical strain $\mathbf{E}=\mathbb{E}(\mathbf{F})$ imposed by a traction on the boundary. The pictorial representation of $\overline{\mathbf{Q}}$ as a function of $\mathbf{E}$ is called a phase diagram. Though this is defined as a map of solutions to a very particular boundary value problem, the phase diagram yields in fact qualitative information on the minimizers of $\mathcal{F}_{X, \varepsilon}^{\Gamma_{D}, g}$ even in the presence of more general boundary conditions, provided that we consider the behavior of solutions sufficiently far away from the boundary. The phase diagrams for all the models considered in this paper are presented and discussed in detail in [8, section 5]. Again, an analogous construction holds also for the case of compressible elastomers.

\footnotetext{
${ }^{2}$ Again, we identify a constant matrix $\mathbf{E}(x) \in L^{2}\left(\Omega, \mathbb{M}_{s y m}^{3 \times 3}\right)$ with a matrix $\mathbf{E} \in \mathbb{M}_{s y m}^{3 \times 3}$.
} 
Acknowledgment. The results contained in this paper have been obtained while the author was a Ph.D. student at Sissa.

\section{REFERENCES}

[1] V. Agostiniani and A. DeSimone, Gamma-convergence of energies for nematic elastomers in the small strain limit, Contin. Mech. Thermodyn., 23 (2011), pp. 257-274.

[2] J.M. BAll AND A. ZARnescu, Orientable and non-orientable director fields for liquid crystals, Proc. Appl. Math. Mech., 7 (2007), 1050701.

[3] P. Bladon, E.M. Terentuev, and M. Warner, Transitions and instabilities in liquid crystal elastomers, Phys. Rev. E, 47 (1993), pp. 3838-3840.

[4] A. Braides, Gamma-Convergence for Beginners, Oxford University Press, Oxford, 2002.

[5] A. Braides, Relaxation of functionals with constraints on the divergence, Ann. Univ. Ferrara Sez. VII (N.S.), 33 (1987), pp. 157-177.

[6] H. Brezis, Analyse fonctionnelle théorie et applications, Masson, Paris, 1983.

[7] P. Cesana, Relaxation of multi-well energies in linearized elasticity and applications to nematic elastomers, Arch. Ration. Mech. Anal., 197 (2010), pp. 903-923.

[8] P. Cesana And A. DeSimone, Strain-order coupling in liquid crystal elastomers: Equilibrium configurations, Math. Models Methods Appl. Sci., 4 (2009), pp. 601-630.

[9] P. Cesana And A. DeSimone, Quasiconvex envelopes of energies for nematic elastomers in the small strain regime and applications, J. Mech. Phys. Solids, 59 (2011), pp. 787-803.

[10] P.G. Ciarlet, Mathematical Elasticity, Vol. 1, Elsevier Science Publishers B.V., NorthHolland, Amsterdam, 1988.

[11] M. Cicalese, A. DeSimone, And C.I. Zeppieri, Discrete-to-continuum limits for strainalignment-coupled systems: Magnetostrictive solids, ferroelectric crystals, and nematic elastomers, Netw. Heterog. Media, 4 (2009), pp. 667-708.

[12] S. Conti, A. DeSimone, And G. Dolzmann, Semi-soft elasticity and director reorientation in stretched sheets of nematic elastomers, Phys. Rev. E, 66 (2002), 061710.

[13] S. Conti, A. DeSimone, And G. Dolzmann, Soft elastic response of stretched sheets of nematic elastomers: A numerical study, J. Mech. Phys. Solids, 50 (2002), pp. 1431-1451.

[14] G. Dal Maso, An Introduction to Gamma-Convergence, Birkhäuser, Boston, 1993.

[15] P.G. De Gennes and J. Prost, The Physics of Liquid Crystals, 2nd ed., Clarendon Press, Oxford, 1993.

[16] A. DeSimone, Energy minimizers for large ferromagnetic bodies, Arch. Ration. Mech. Anal., 125 (1993), pp. 99-143.

[17] A. DeSimone, A. DiCarlo, And L. Teresi, Critical voltages and blocking stresses in nematic gels, Eur. Phys. J. E, 24 (2007), pp. 303-310.

[18] A. DeSimone and G. Dolzmann, Macroscopic response of nematic elastomers via relaxation of a class of SO(3)-invariant energies, Arch. Ration. Mech. Anal., 161 (2002), pp. 181-204.

[19] A. DeSimone And G. Dolzmann, Stripe-domains in nematic elastomers: Old and new, in Modeling of Soft Matter, IMA Vol. Math. Appl. 141, Springer, New York, 2005, pp. 189203.

[20] A. DeSimone and L. Teresi, Elastic energies for nematic elastomers, Eur. Phys. J. E, 29 (2009), pp. 191-204.

[21] J.L. ERICKSEN, Liquid crystals with variable degree of orientation, Arch. Ration. Mech. Anal., 113 (1991), pp. 97-120.

[22] I. Fonseca And G. Leoni, Modern Methods in the Calculus of Variations: $L^{p}$ Spaces, Springer, New York, 2007.

[23] F.C. Frank, P.J. Wojtowicz, and P. Sheng, On the theory of liquid crystals, Discuss. Faraday Soc., 25 (1958), pp. 19-28.

[24] G.P. GaLdi, An Introduction to the Mathematical Theory of the Navier-Stokes Equations, Vol. 1, Springer, New York, 1994.

[25] J.B. Hiriart-Urruty and C. Lemaréchal, Convex Analysis and Minimization Algorithms, Vol. 1, Springer, New York, 1993.

[26] L. Longa, D. Monselesan, and H.R. Trebin, An extension of the Landau-Ginzburg-de Gennes theory for liquid crystals, Liquid Crystals, 2 (1987), pp. 769-796.

[27] S. MüLLER, Variational models for microstructure and phase transitions, in Calculus of Variation and Geometric Evolution Problems, Cetraro 1996, F. Bethuel, G. Huisken, S. Müller, K. Steffen, S. Hildebrandt, and M. Strüwe, eds., Lecture Notes in Math. 1713, Springer, New York, 1999, pp. 85-210.

[28] E.G. Virga, Variational Theories for Liquid Crystals, Chapman \& Hall, London, 1994.

[29] M. Warner and E.M. Terentjev, Liquid Crystal Elastomers, Clarendon Press, Oxford, 2003.

Copyright (c) by SIAM. Unauthorized reproduction of this article is prohibited. 